\title{
SINTOMATOLOGÍA DEPRESIVA EN ESCOLARES DE 12 A 16 AÑOS Y SU RELACIÓN CON LAS DISTORSIONES COGNITIVAS
}

\author{
MIGUEL A. CARRASCO ${ }^{1}$, VICTORIA DEL BARRIO ${ }^{1}$ y JUAN F. RODRÍGUEZ ${ }^{2}$ \\ ${ }^{1}$ Facultad de Psicología, Universidad Nacional de Educación a Distancia \\ ${ }^{2}$ Facultad de Psicología, Universidad de Sevilla
}

(Recibido el 28 de enero de 1999)

\begin{abstract}
Los principales objetivos de esta investigación han sido el estudio de la presencia de sintomatología depresiva y su relación con las distorsiones cognitivas en escolares adolescentes. Los instrumentos utilizados han sido el inventario CDI (Kovacs, 1992), el inventario de depresión PNID de nominación de iguales (Lefkowitz y Tesiny, 1981) y el cuestionario de distorsiones cognitivas CDCN-1 (Bas, 1987). Sobre una muestra representativa de la ciudad de Sevilla de 908 jóvenes y de edades comprendidas entre 12 y 16 años, se ha hallado una presencia de sintomatología depresiva del $11^{\prime} 78 \%$, con una tendencia lineal ascendente respecto de la edad y con puntuaciones superiores en el caso de las mujeres. El número de distorsiones cognitivas se ha relacionado positivamente con la presencia de esta sintomatología, mostrando ser uno de los principales predictores de las puntuaciones en el CDI. La sintomatología depresiva guarda relación con los momentos más importantes del desarrollo cognoscitivo en el joven, apreciándose un aumento de distorsiones cognitivas a los 13 años de edad que se mantiene constante después. El CDCN-1 se estima como un adecuado complemento del CDI, al contrario que el PNID, probablemente por el proceso de internalización de las manifestaciones depresivas. No se puede otorgar a los sesgos cognitivos un papel causal sobre la depresión, pero se sugiere que la coincidencia en el CDI y el CDCN-1 de puntuaciones elevadas es un indicio de un elevado riesgo (vulnerabilidad) para desarrollar un primer episodio depresivo.
\end{abstract}

Palabras clave: Sintomatología depresiva, adolescencia, distorsión cognitiva, vulnerabilidad. Depressive symptomatology in schooles of 12 to 16 years and their relationship with
cognitive distortions

The aims of this investigation have been the study of the presence of depressive symptomatology and their relationship with cognitive distortions in adolescent scholars. Children's Depression Inventory (CDI, Kovacs, 1992), Peer Nomination Inventory of Depression (PNID, Lefkowitz y Tesiny, 1981) and Children's Cognitive Distortions Questionnaire (CDCN-1, Bas, 1987) have been administered on a representative sample of 908 Sevillian adolescents (aged 12 to 16). The results revealed an $11^{\prime} 78 \%$ of depressive symptomatology with an upward lineal tendency regarding the age and with superior scores in the case of women. The number of cognitive distortions has been related positively with the presence of this symptomatology. This point shows to be one of the main predictors of the punctuations in CDI. The depressive symptomatology keeps relation to the most important moments in the youth's cognitive development. An increase of cognitive distortions has been appreciated on aged 13. Later on, this one stays constant. CDCN-1 is considered as an appropriate complement of CDI, on the contrary that PNID, probably for the process of internalization of depressive manifestations. You cannot grant a causal role on depression to the cognitive biases. It is suggested that the coincidence between $\mathrm{CDI}$ and $\mathrm{CDCN}-1$ about raised scores is a sign of a high risk (vulnerability) to develop a first depressive episode.

Key words: Depressive symptomatology, adolescence, cognitive distortion, vulnerability.

Agradecimientos. Nuestro agradecimiento a los
alumnos que han ayudado de forma inestimable en la
recogida y corrección del material manejado en esta
investigación: M. Mar Benítez; M. Carmen Catalán;
Luis Salvador García; Antonia Gómez; Isabel Gutié-
rrez; Isaac Martín; Jesús Ortega; Carolina Otón; Isabel
Rojas; Eneka Sagastagoitia; Isabel Sánchez-Barriga; M
del Mar Tirado; M Carmen Troya. Quisiéramos tam- bién agradecer al Gabinete Psicológico INTELEN la colaboración prestada que ha hecho posible la elaboración y preparación del trabajo realizado.

Correspondencia: Juan F. Rodríguez Testal, Dpto. de Psiquiatría, Personalidad, Evaluación y Tratamiento Psicológicos, Facultad de Psicología, Universidad de Sevilla, Avda. San Francisco Javier s/n, 41005 Sevilla. Tfnos.: 954557802 / 954280 309. Fax: 954557807. 


\section{INTRODUCCIÓN}

Actualmente se reconocen las particularidades sintomáticas de las depresiones entre niños y jóvenes, así como la necesidad de encuadrarlas de forma más precisa dentro de una perspectiva evolutiva (Bas, 1996; Doménech, 1988; Kovacs, 1985). La posibilidad de diagnosticar esta alteración se acepta para edades muy tempranas, como los casos de estudios de depresión en preescolares (Doménech y Moreno, 1985; Kashani, Holcomb y Orvaschel, 1986; Kashani y Ray, 1987) cuando antaño se negaba incluso su existencia (del Barrio y Moreno, 1996), del mismo modo que en las primeras etapas escolares y, con mayor propiedad, dentro del periodo adolescente. Este último caso se constituye, precisamente, en una de las poblaciones de mayor incidencia de sintomatología depresiva (del Barrio, 1997) y la segunda con el mayor riesgo de suicidio, después de los mayores de 65 años (Bas, 1996).

La necesidad de ahondar en el conocimiento de esta realidad se hace patente pues es difícil determinar con exactitud los mecanismos etiológicos, los factores favorecedores o precipitantes y los mantenedores de la sintomatología y/o de los trastornos del estado de ánimo. Por tanto, y como base fundamental para el establecimiento de medidas preventivas (y quizá terapéuticas específicas), es necesario precisar cómo se distribuye este problema en la población seleccionada, conocer qué prevalencia presenta y en función de qué variables ambientales y personales se manifiesta (Doménech, 1988). Sin el conocimiento de estos datos difícilmente se puede abordar el esclarecimiento de los factores de riesgo asociados en esta población, los elementos causales en su dinámica y la intervención sobre los mismos, preferentemente desde una óptica preventiva y un talante educativo que facilite a los jovenes al afron- tamiento de sus dificultades. Creemos que lo referido justifica la importancia de los estudios encaminados a la obtención de información epidemiológica (del síntoma o del trastorno depresivo) más una permanente actualización de los mismos que garantice un mejor aprovechamiento de los recursos sanitarios. Al tiempo, el logro de las metas mencionadas representa un ambicioso proyecto del que el presente trabajo no es más que el primer paso, fundamentalmente centrado en la sintomatología depresiva y en algunas características alrededor de la misma, principalmente el interés de diferentes instrumentos y el relieve que cobran las distorsiones cognitivas.

La depresión infantil y juvenil, a nivel sindrómico, es una alteración del humor cuyos síntomas se extienden en diferentes áreas (APA, 1995; del Barrio, 1990; 1997): emocionales (tristeza, pérdida del tono hedónico, irritabilidad, ...), motoras (baja o alta actividad, inexpresividad, ...), cognitivas (desesperanza, culpa, pérdida de concentración y memoria, ...), sociales (aislamiento, soledad, rechazo, ...), conductuales (protestas, riñas, escapismo, ...) y somáticas (enuresis, fatiga, pérdida de peso, ...). De todos estos síntomas, los más consensuados han sido la baja autoestima, alteraciones del sueño, disminución de la socialización, alteraciones del apetito y el peso, agitación motora, agresión, disforia e ideación suicida (Del Barrio, 1990; McNeil y Harsany, 1989).

Aunque la sintomatología depresiva infantil y adolescente es similar y equiparable a la adulta, es reconocido que adquiere peculiaridades evolutivas dependientes de la edad, o mejor, del estadio del desarrollo (Carlson y Kashani, 1988; Weiss, Weisz, Politano, Carey, Nelson y Finch, 1992), evolucionando desde una sintomatología exteriorizada, fundamentalmente motora y psicosomática propia de los niños menores de 6 
años (Doménech y Moreno, 1995; Kashani et al., 1986; Kashani y Carlson, 1987; Kashani y Ray, 1987; Kashani, Ray, Gabrielle y Carlson, 1984), a una sintomatología más interiorizada similar a la de los adultos que claramente se instaura en la adolescencia (Bas, 1996; Cantwell, y Carlson, 1987; Carlson y Kashani, 1988; Lewinsohn, Roberts, Seeley, Rohde, Gotlib y Hops, 1994). Además, en los niños se observa tendencia al aislamiento, elevada ansiedad, irritabilidad, comportamiento perturbador, problemas de atención y de rendimiento escolar. Los adolescentes presentan también menoscabo en la atención y ansiedad, pero los problemas disociales pueden ser de bastante importancia junto al abuso de sustancias y trastornos de la alimentación (APA, 1995; Baker, 1991).

Ante estas dificultades de enfoque de los síntomas relevantes para estas diferentes edades, a veces frecuentes entre adolescentes sin que representen un cuadro clínico de depresión, del Barrio y Moreno (1996) proponen una definición integradora de la depresión infantil, es decir, dentro del nivel trastorno, como un «cambio persistente en la conducta de un niño que consiste en un descenso en la capacidad de disfrutar de los acontecimientos, comunicar con los demás, rendir escolarmente y acompañado de acciones de protesta plural» (pág. 135).

Los aspectos metodológicos de la evaluación de la depresión infanto-juvenil, entre los que han destacado el uso de diversas fuentes de información, la utilización de distintos instrumentos, los rangos de edad seleccionados, el empleo de diferentes muestras clínicas o subclínicas (del Barrio, 1990; Fleing y Offord, 1990), han dificultado clarificar los datos epidemiológicos de esta alteración, hecho que ha dado lugar a resultados muy dispares que han oscilado, inicialmente, de un 0 ' $14 \%$ de niños de 10 y 11 años y del $1 ' 5 \%$ para los de 14 a 15 años (Rutter,
Tizard y Whitmore, 1970), hasta un $60 \%$ (Carlson y Cantwell, 1980). La depuración de los aspectos metodológicos, han permitido, en el momento actual, establecer cierto consenso dentro de un margen de variabilidad menor, en general por debajo del 3\% (Fleing y Offord, 1990), fluctuando entre el 1 y el $4 \%$ de Trastorno Depresivo Mayor en la primera infancia, de un 2 a un $9 \%$ en la segunda infancia y de un 2 a un $15 \%$ de este trastorno en la adolescencia, tanto en población española como extranjera (revisiones de Anderson, Williams, McGee y Silva, 1987; Cicchetti y Toth, 1998; Cohen, Cohen, Kasen, Velez, et al., 1993; Costello, Costello, Edelbrock, Burns, et al., 1988; del Barrio, 1997; del Barrio, Moreno y López, 1997; McGee, Feehan, Williams, Partridge, et al., 1990).

Centrándonos en el ámbito científico que nos ocupa, Polaino-Lorente y Doménech (1988) llevaron a cabo un trabajo de investigación a escala nacional sobre 6.436 escolares de 8 a 11 años de edad que incluía a la ciudad de Sevilla (entorno en el que se enmarca este trabajo). En dicho estudio se estima una prevalencia total de Trastorno Depresivo Mayor del $1{ }^{\prime} 8 \%$ ( 2 ' $8 \%$ en niñas y 0 ' $9 \%$ en niños) y del 6' $4 \%$ para el Trastorno Distímico por medio de un procedimiento depurado de selección de la muestra, más la entrevista clínica CDRS-R. Doménech (1988) en su revisión sobre la bibliografía de depresión infantil, concluye que la incidencia del Trastorno Depresivo Mayor en población no clínica está por debajo del $2-3 \%$. En este sentido, también Ezpeleta (1988) en la ciudad de Barcelona, utilizando criterios DSM-III en niños de EGB coincide en señalar un 1'7\% de Depresión Mayor y 9'3\% de Trastorno Distímico. Posteriormente alcanza cifras muy inferiores a las citadas pues de una muestra de niños y niñas de 8 a 13 años $(N=1.121)$, tras la fase de cribado evaluados por los profesores $(n=76)$, registra 4 casos de Trastor- 
no Depresivo Mayor y 11 de Trastorno Distímico (Ezpeleta, Polaino-Lorente, Doménech y Doménech, 1990).

En cambio, si se estudia en población pediátrica, con patología orgánica asociada, la presencia oscila de un $8-10 \%$ a un $30 \%$, variaciones atribuibles o dependientes del tipo de afección somática asociada. De forma esperable, estos datos de presencia de trastornos del estado de ánimo se incrementan cuando se trabaja sobre población clínica. De este modo, con muestras españolas Bragado, Carrasco, Sánchez, Bersabe, Loriga y Monsalve (1995) con 236 sujetos de 6 a 17 años, encuentran un $7{ }^{\prime} 2 \%$ de depresión a través de la entrevista diagnóstica DICA-R para niños y adolescentes. También con población clínica pero sobre sintomatología depresiva, López Soler y López Pina (1998), encuentran en 182 niños entre 6 y 11 años un 15\% de chicos y un $17 \%$ de chicas con indicadores significativos evaluados a través de un inventario clínico para padres, adaptado del CBC de Achenbach y Edelbrock y de la escala para padres de Conners. De otros estudios llevados a cabo en población general, Polaino-Lorente (1995) ha señalado el incremento de esta alteración con la edad y el descenso de la prevalencia desde la etapa adolescente, escolar a la preescolar, siendo estimada, por diferentes autores, en este último caso, entre el 0 '9\% y el $1 \%$ (Kashani, et al., 1986; Kashani y Carlson, 1987).

Estos datos de prevalencia, obtenidos en la población infantil y adolescente ponen de relieve el papel mediador destacado sobre la sintomatología y el nivel sindrómico depresivos de dos variables sociodemográficas: la edad y el sexo. En relación con la edad, la sintomatología depresiva y el diagnóstico de trastornos del estado de ánimo parecen aumentar a medida que el niño crece, llegando a su nivel más elevado en la adolescencia (Kashani et al., 1986; Kashani y Carlson,
1987; Mestre, Frías, García Ros y del Barrio, 1992; Nogueira y Monreal, 1990; Polaino-Lorente, 1995; Polaino-Lorente y Doménech, 1988; 1990; Polaino-Lorente y García-Villamisar, 1993; Rutter et al., 1970), sobre todo para la sintomatología (del Barrio y Moreno, 1996; Fleing y Offord, 1990) y principalmente entre las mujeres (Cohen et al., 1993). Como se ha constatado en población española, Nogueira y Monreal (1990) entre niños de 8 a 11 años y Mestre et al. (1992) con niños de edades de 9 a 15 años, los sujetos de mayor edad informaron significativamente de más sintomatología depresiva. En la misma línea, se ha concluido recientemente en el trabajo de Hankin, Abramson, Moffitt, Silva y McGee (1998), quienes hallan las mayores puntuaciones en depresión y la aparición de casos nuevos en los años de transición de la adolescencia media a la adolescencia tardía en un estudio longitudinal de 10 años de duración, con una población que osciló en número entre 792 y 961 , desde los 11 años de los sujetos inicialmente seleccionados hasta los 21 años al finalizar el estudio.

Con relación al sexo, diferentes estudios han coincidido en la superioridad del género femenino en lo que a sintomatología depresiva se refiere (Angold y Rutter, 1992; Nolen-Hoeksema y Girgus, 1994), pero también cuando se alude al nivel trastorno (APA, 1995; Boyd y Weissman, 1985; Cross-National Collaborative Group, 1992; Friedman y Thase, 1995; Weissman, Bland, Joyce, Newman, Wells y Wittchen, 1993). Sin embargo, en población infantil, estas diferencias no parecen emerger hasta llegada la adolescencia (Cohen et al., 1993; Frías, Mestre, del Barrio y García Ros, 1992; Hankin et al., 1998; Joiner, Blalock y Wagner, 1999; Nolen-Hoeksema, y Girgus, 1994), aunque algunos estudios apuntan a que estas diferencias de género en adolescentes son exclusivas de la población clínica y 
no de la población general (Compas, Oppedisano, Connor, Gerhardt, Hinden, Achenbach y Hammen, 1997) y donde las diferencias de sexo en magnitud son muy pequeñas (Lewinsohn, Hops, Roberts, Seeley y Andrews, 1993; Petersen, Sarigiani y Kennedy, 1991).

Las diferencias que conectan la edad y al sexo, han sido explicadas a través de diversos modelos (Hankin, et al., 1998; Nolen-Hoeksema y Girgus, 1994) atendido tanto a elementos de naturaleza biológica ligados a la adolescencia, a aspectos psicosociales como el apoyo social o a la presencia de sucesos estresantes que parecen ejercer un efecto diferencial en varones y mujeres y de extraordinario relieve en comparación con otros momentos vitales (Lewinsohn, Gotlib y Seeley, 1997).

La aparición de alteraciones depresivas en los niños y adolescentes ha sido explicada, desde diferentes perspectivas, entre las que destaca el modelo cognitivo y concretamente el procesamiento de la información, de forma similar a como se ha hecho en los adultos (Dodge, 1993; Gladstone y Kaslow, 1995; Kazdin, Rodgers y Colbus, 1986; Seligman, Kaslow, Alloy, Peterson, Tanenbaum y Abramson, 1984; Stark, Humphrey, Laurent, Livingston y Christopher, 1993). El procesamiento sesgado de la información en relación con los eventos vividos por el niño, como los errores en cada uno de los pasos del procesamiento (atención selectiva de información negativa, atribuciones de causalidad, errores cognitivos, acceso a respuestas desviadas y poco asertivas, evaluaciones y expectativas más negativas) pueden llevar al niño al desarrollo de una alteración afectiva (Dodge, 1993). Aaron Beck y su equipo de la Universidad de Pensilvania (Filadelfia, USA) (Beck, Rush, Shaw y Emery, 1983) refieren distintas estructuras de conocimiento (esquemas) que permiten operaciones y proposiciones cognitivas sesgadas (dis- torsiones cognitivas o actitudes disfuncionales, por ejemplo) y ciertos productos (como los pensamientos automáticos) adquiridos en la historia del sujeto que genera un estilo en el procesamiento primitivo y negativo (extremista, arbitrario, absolutista, sentencioso), aunque no se les puede conceder un papel exclusivamente causal en la depresión (Harrison, Beck y Buceta, 1984). Los pensamientos no causan las modificaciones neuroquímicas ni al revés, son los mismos procesos desde ópticas diferentes como señaló acertadamente Beck (1988; 1991; 1997; Clark y Beck, 1997), debiendo ocupar un puesto destacado en la consideración de los síntomas de la depresión. Por tanto, estos elementos cognitivos son entrelazados con componentes biológicos y sociales que conducen al niño, como ha indicado Cicchetti y Toth (1998), a la formación de una organización depresógena que le hace vulnerable al desarrollo de sintomatología depresiva.

Stark et al. (1993) analizan la tríada cognitiva de Beck y los pensamientos automáticos en un total de 59 niños de los cuales 16 habian sido diagnosticados con trastornos depresivos, 11 con desórdenes de ansiedad y 16 sujetos de control. Las cogniciones depresógenas, tales como percepciones y pensamientos negativos sobre el yo, el mundo y el futuro, discriminaban entre los grupos clínicos y el grupo control, pero especialmente entre los niños deprimidos y ansiosos.

Asarnow y Bates (1988) destacaron la relevancia de los pensamientos o cogniciones negativas como las atribuciones en la depresión de los adolescentes. Asimismo, Lewinsohn et al. (1997) estudiaron diversas cogniciones como autorreforzamiento, actitudes disfuncionales, control percibido, autoconciencia y estilo atribucional en tres grupos de adolescentes, 48 deprimidos, 92 con otras alteraciones psicopatológicas distintas a la depresión y 1.079 sujetos testigos. Las cogniciones 
negativas, especialmente el estilo atribucional depresógeno, aparecieron fuertemente asociadas a los sujetos deprimidos, no obstante, estas variables no fueron específicas de este grupo de sujetos. El estilo atribucional interno, estable y global para los eventos negativos y externo, inestable y específico para los eventos positivos ha sido claramente relacionado con la sintomatología depresiva en niños en un metanálisis sobre 28 estudios, realizado por Gladstone y Kaslow (1995).

Otro grupo de representaciones mentales asociadas con la depresión infantil ha sido el relativo a los errores cognitivos o distorsiones (Haley, Fine, Marriage, Moretti y Freeman, 1985; Leitenberg, Yost y Carroll-Wilson, 1986) con las que los niños interpretan y valoran lo que les acontece. En este sentido, Haley et al. (1985) relacionan la presencia de pensamientos distorsionados con los trastornos del estado de ánimo en estas edades y Leitenberg et al. (1986) encuentran correlaciones significativas entre los sesgos cognitivos de sobregeneralización y catastrofismo con la depresión en niños y adolescentes.

Al hilo de lo indicado y siguiendo a Karel (1997), puede observarse en los comienzos de la edad adulta la mayor importancia en la consideración de una diátesis cognitiva frente a la biológica e incluso de los eventos vitales en el origen de la depresión. En esta dirección apuntan los últimos trabajos de Abramson, Alloy, Metalsky, Joiner y Sandín, B. (1997) acerca de una vulnerabilidad biocognoscitiva $y$, más recientemente, Abramson, Alloy, Hogan, Whitehouse et al. (1999), en donde se aportan evidencias de la vulnerabilidad cognitiva a la depresión clínica (así como para la sintomatología depresiva, la denominada depresión por desesperanza y el suicidio), traducidas en forma de atribuciones negativas, procesamiento autorreferente y otorgando, además, valor a las actitudes disfuncionales de Beck. El origen podría emerger desde la psicopatología de los padres (modelado, retroalimentación y eventos negativos), el abuso o maltrato y la negligencia consecuencia de anteriores episodios depresivos.

En todo caso, parece relevante la consideración de los elementos cognitivos como posibles elementos predisponentes de la depresión y su enraizamiento en muchas experiencias o situaciones acaecidas en niños y jóvenes. El análisis de sus diferentes componentes, como en el presente trabajo, el buceo a propósito de las distorsiones cognitivas, permitiría aportar información en esta población en equivalencia con los adultos acerca del modelo de vulnerabilidad cognitiva a la depresión, precisamente en el momento evolutivo en el que toma cuerpo o se consolida la estructura mental de una persona. No obstante, aunque es difícil determinar si realmente estos componentes son causas, consecuencias o concomitantes (Polaino-Lorente, 1996), no es menos cierto que establecer su papel como predisponentes o como factores de riesgo tienen una significación terapéutica y preventiva (Abramson et al., 1999).

En suma, se han planteado las dificultades en establecer datos precisos o definitivos acerca de la prevalencia de los trastornos del estado de ánimo en edades infanto-juveniles. En parte, por las diferencias metodológicas entre las investigaciones pero, especialmente, por el protagonismo de la variable referente al momento evolutivo del sujeto en cuestión. Es evidente que el porcentaje de sintomatología depresiva supera la prevalencia de los trastornos del humor en sentido estricto, siendo este comentario más cierto para la etapa adolescente $\mathrm{y}$, dentro de este último rango de edad, para el caso de las mujeres. Partiendo de esta realidad muchos trabajos se han centrado en el análisis de la sintomatología depresiva, mayoritariamente a través de 
autoinformes, ya que permiten trabajar con muestras más amplias y poner a prueba más fácilmente sus hipótesis (del Barrio y Moreno, 1996), aspectos menos factibles cuando se consideran exclusivamente los trastornos depresivos. Consideramos que aparte de las ventajas que supone para la investigación asumir una perspectiva dimensional, aludir a la sintomatología depresiva representa un problema de salud por el que también se consulta o pide ayuda, especialmente cuando las manifestaciones son abundantes, aunque no sugieran un trastorno desde un punto de vista formal.

En este trabajo hemos pretendido estudiar y actualizar la presencia de sintomatología depresiva en la población infanto-juvenil de la ciudad de Sevilla. Dado que se trata de un estudio transversal y de naturaleza exploratoria, no hemos tratado de proporcionar diagnósticos de trastornos del estado de ánimo o de selección de "casos" y, como se ha mencionado en el párrafo anterior, disponer de una amplia muestra permitiría sondear la presencia de distorsiones cognitivas y su posible conexión con la sintomatología depresiva. Por consiguiente, no se trata de asumir relaciones etiológicas, sino de aproximarse al fenómeno depresivo desde la consideración de un proceso cognitivo escasamente referido en la literatura científica española.

\section{OBJETIVOS}

1. Determinar la presencia de sintomatología depresiva entre los jóvenes de 12 a 16 años, permitiendo actualizar la información existente al respecto.

2. Relacionar las diferentes fuentes de medida para la sintomatología depresiva.

3. Analizar la relación entre esta sintomatología depresiva con las distorsiones cognitivas presentadas en las res- puestas dadas por los jóvenes estudiados.

\section{MÉTODO}

\section{Sujetos}

La muestra definitiva quedó conformada por 908 personas, 466 varones ( $51^{\prime} 3 \%$ ) y 442 mujeres ( 48 ' $7 \%$ ), con edades comprendidas entre los 12 a 16 años, escolares de Sevilla capital y pertenecientes a 11 centros ubicados en la zona centro de la ciudad ( 3 centros), zona media ( 4 centros) y de la periferia (4 centros).

\section{Instrumentos}

Los instrumentos utilizados en este estudio y algunas de sus características son:

- Inventario CDI de depresión (Children's Depression Inventory) (Kovacs, 1992). Consta de 27 ítemes, cada uno de los cuales está graduado de 0 a 2 según la intensidad de la sintomatología depresiva. El contenido de cada elemento versa sobre aspectos cognitivos, afectivos, fisiológicos y motivacionales. Su aplicación es válida para sujetos de 6 a 17 años. Se utilizó la versión de Polaino-Lorente (1988) con el punto de corte en 19 o más respuestas significativas.

- Inventario PNID (Peer Nomination Inventory of Depression) (Lefkowitz y Tesiny, 1981). Este instrumento evalúa la depresión tomando como informadores externos a los iguales, de manera que cada integrante de una clase es preguntado sobre qué compañeros exhiben determinadas conductas propias de la sintomatología depresivas (las más externalizadas). Consta de 23 ítemes, 4 introductorios para la adap- 
tación a la prueba y 19 específicos repartidos en tres subescalas: Depresión (13 preguntas, por ejemplo, $\mathrm{n}^{2} 11$ : “Quién llora muchas veces?»), Felicidad (4 preguntas, por ejemplo, $n^{2} 13$ : "¿A quién le gusta hacer un montón de cosas?») y Popularidad ( 2 ítemes, por ejemplo, $n^{\circ} 22$ : “¿A quién te gustaría tener sentado a tu lado en clase?»). Se ha aplicado la versión de Polaino-Lorente (1988) y Ezpeleta et al. (1990), estableciendo 4 puntos como línea de corte.

- Cuestionario de Distorsiones Cognitivas para Niños, CDCN-1 (Bas, 1987b; Bas y Andrés, 1994). Esta prueba consta de 11 ítemes, cada uno de los cuales expresa una distorsión cognitiva (sobregeneralización, polarización, inferencia arbitraria, pensamiento dicotómico, ...) a las que el sujeto ha de contestar si tiene habitualmente este pensamiento, por ejemplo, ítem 2: "cuando pierdo en un juego pienso que nunca ganaré» (Bas, 1987 a,b). La corrección se lleva a cabo sumando simplemente las respuestas afirmativas. El punto de corte establecido es de 7 o más respuestas positivas.

\section{Diseño, Variables y Condiciones de Control}

Se utilizó un diseño correlacional de comparación de grupos dentro de un método transversal y en función del sexo y la edad. Las variables predictivas fueron el sexo (valor de 1 para varones y 2 para las mujeres) y la edad cronológica en años, determinada por el propio individuo en el momento de la evaluación, tomada como variable predictiva cualitativa (5 categorías, una por edad) o de forma cuantitativa. Las variables criterio fueron los resultados o puntuaciones de cada una de las pruebas administradas en el único momento de medida establecido.
Para mantener el anonimato de los participantes, en la mayoría de los centros se obtuvo una lista con los alumnos y sus números en el aula (en los casos contrarios se elaboró al efecto). Además, en la aplicación del cuestionario PNID, gracias a este recurso, se instruyó a los participantes para que preguntaran en privado, en caso de duda, a uno de los examinadores el número o el nombre de las personas nominadas.

\section{Procedimiento}

Para la determinación de la muestra representativa utilizada se partió del censo correspondiente a la ciudad de Sevilla (IAE, 1992). Aunque la referencia de los datos no estaba completamente actualizada se estimó el grupo poblacional correspondiente que, en el comienzo del estudio, Octubre de 1996, tendría entre 12 y 16 años.

El número de participantes necesarios para cada grupo de edad se llevó a cabo conociendo el porcentaje poblacional, asumiendo un error predeterminado del $7 \%$ y para un nivel de confianza del $95 \%$. Se estableció, por tanto un tamaño muestral de 970 personas (496 varones, $51^{\prime} 1 \%$ y 474 mujeres, $48^{\prime} 8 \%$ ). Si bien es cierto que el volumen de sujetos era grande, una de las pretensiones del estudio era lograr, para cada grupo de edad, una proporción realmente representativa de varones y mujeres, haciendo que el total de personas, según el grupo de edad que se tratara, oscilara entre 94 y 101 sujetos.

Posteriormente, se dividió a la ciudad en tres áreas o núcleos poblacionales, tomando como base el punto considerado como centro histórico. La distribución de estas áreas se hizo con forma de anillos circunscritos, cada uno con dos kilometros de radio. 
Partiendo de una relación de centros públicos y concertados de Sevilla y su provincia de la Consejería de Educación de la Junta de Andalucía se escogieron aquellos ubicados en cada uno de los anillos descritos que dividían la ciudad. Se tomaron tres para la zona centro, y cuatro para el medio y la periferia (debido a la mayor concentración de unidades escolares), quedando constituido el conjunto por un total de 11 colegios e institutos (9 centros públicos y 2 concertados). A continuación, se contactó con la dirección de cada unidad escolar presentando el proyecto de trabajo que incluía una autorización dirigida a los padres (por si tenían algún tipo de inconveniente para que su hijo/a participara en la evaluación). Seguidamente, y tras dejar una semana para que los padres dieran respuesta a la petición realizada, se llevó a cabo la administración de las pruebas.

Debido a que la capacidad de alumnado de los centros y los niveles académicos que abarcaban diferían notablemente unos de otros, se solicitó a los centros la participación de dos aulas correspondientes a cada grupo de edad, con independencia de que en algunos estuviera vigente todavía el anterior sistema educativo o, por el contrario, se estuviera aplicando el correspondiente a la reforma. Aunque eso dio lugar a una extraordinaria cantidad de personas participantes, era esperable diversas fuentes de pérdidas de datos: sujetos cuya cumplimentación no fuese completa, personas que superaran la edad requerida, alumnos de integración (se descartaron para lograr una mayor homogeneidad en la muestra), participantes de los que se tuviera constancia de que estuvieran en tratamiento psicológico o psiquiátrico (por medio del propio sujeto, los padres o el orientador del centro), absentismo o negativa a realizar las pruebas. Con todo, para evitar la saturación de personas de un mismo colegio o instituto, ya que la capacidad de las aulas difería ampliamente de unas unidades escolares a otras, se tomó el centro más pequeño en capacidad de alumnado como unidad. Después se dividió el resto de los participantes de los demás centros en función de esta unidad, de manera que se fueron escogiendo al azar las sucesivas unidades hasta completar los 908 sujetos constituyentes de la muestra definitiva. Como se apreciará, no se lograron los 970 estimados inicialmente pues el grupo de edad correspondiente a 16 años, no alcanzó los 196 sujetos requeridos aunque el porcentaje poblacional en función del sexo se cumplíó.

La administración de las pruebas (CDI, PNID y CDCN-1) fue colectiva y realizada en una ocasión, con duración aproximada de una hora $u$ hora y cuarto. Las horas de la realización de la evaluación fue diferente según cada centro, ya que se llevó a cabo en función de las posibilidades de cada uno de ellos. Se realizó una presentación ante los alumnos, se separaron los casos que no contaran con el permiso de los padres y se explicó que la finalidad del estudio versaba en conocer cómo piensan los alumnos sobre una serie de temas y sobre las características personales de cada uno. Los datos se recabaron en el lapso de tiempo entre Noviembre de 1996 y Febrero de 1997. Posteriormente, se devolvieron los resultados en un breve informe a la dirección de cada centro.

El procedimiento estadístico se basó en pruebas descriptivas (frecuencias, porcentajes, correlaciones de Pearson a dos colas para $p<0$ '01), inferenciales como el análisis de la varianza unidireccional $(p<0$ '05), incluyendo el análisis de la homogeneidad de la varianza de Bartlett $(p<0$ 05), en su caso, la prueba de rangos de Scheffé $(p<0$ '05), análisis factoriales de la varianza $2 \times 5$ (sexo y edad) y, finalmente, análisis multivariados como el análisis de regresión múltiple por 
pasos ( $p<0$ '05). Los análisis se llevaron a cabo con el paquete estadístico SPSS$\mathrm{PC}+$ versión 4 '0.

\section{RESULTADOS}

Características generales de la muestra

La muestra definitiva compuesta por 908 sujetos presentó frecuencias y porcentajes equivalentes a los estimados como representativos. El porcentaje global de varones y mujeres (52'2 y $47^{\prime} 8$, respectivamente) se aproxima al poblacional (51 y 48'9, respectivamente). El total de sujetos participantes por edad fue siguiente: $192(21$ '1\%) de 12 años, 195 (21'4\%) de 13 años, 192 (21'1\%) de 14 años, $195\left(21^{\prime} 4 \%\right)$ de 15 años y 134 (14'7\%) de 16 años. La media de edad de la población recogida fue de 13 '87 años (1'36 de desviación típica).

\section{Diferencias en función del sexo}

La información descriptiva procedente de las pruebas aplicadas en esta muestra (Tabla 1) muestra que en el inventario CDI la media de depresión en las mujeres es superior al de los varones de forma estadísticamente significativa $(\mathrm{F}=$ $5^{\prime} 220, p=0$ '022), producto que aparece sistemáticamente en los estudios de población adolescente (del Barrio, 1997).
En el cuestionario CDCN-1 la media global de la muestra es de 2'71 puntos, ligeramente inferior a los 2'87 puntos recogidos por Bas y Andrés (1994). En la presente investigación, las mujeres aunque puntúan con mayor promedio no se diferencian significativamente de los varones en el número de distorsiones cognitivas ( $\left.F=0^{\prime} 702, p=0^{\prime} 402\right)$. En el caso del inventario PNID, los varones promediaron más alto en las subescalas de Depresión ( $F=0$ '200, $p=0$ '654) y Popularidad ( $F=0$ '078, $p=0$ '780), al contrario que en la subescala de Felici$\operatorname{dad}\left(F=0^{\prime} 556, p=0^{\prime} 455\right)$. No obstante, las diferencias para las tres subescalas del PNID no fueron estadísticamente significativas en función del sexo.

Estos productos se complementan con el hecho que se obtuvieran buenos resultados en la homogeneidad de la varianza ( $F$ de Bartlett) en función del sexo y para los cuestionarios $\mathrm{CDI}\left(\mathrm{F}=00^{\prime} 710, p=\right.$ 0'400), $\mathrm{CDCN}-1$ ( $\mathrm{F}=0$ '001, $p=0$ '978) y la subescala del PNID de Felicidad $(\mathrm{F}=$ 0'353, $p=0$ '553). En el caso de las subescalas de Depresión y Popularidad, no se obtiene la condición de la homocedasticidad $\left(F=8^{\prime} 695, p=0^{\prime} 003\right.$ y $5^{\prime} 325, p=$ 0'021, respectivamente).

\section{Diferencias en función de la edad}

Las medias y desviaciones tipo de las pruebas aplicadas a toda la muestra

Tabla 1. Medias y desviaciones tipo (entre paréntesis) de las pruebas aplicadas a toda la población

\begin{tabular}{|c|c|c|c|c|c|}
\hline 12 a 16 años & $C D I$ & PNID-D & PNID-F & PNID-P & $C D C N-1^{(1)}$ \\
\hline Total (908) & $11^{\prime} 950\left(5^{\prime} 67\right)$ & 0'440 (0'71) & $0^{\prime} 256\left(0^{\prime} 38\right)$ & 0'195 (0'17) & $2^{\prime} 712\left(1^{\prime} 86\right)$ \\
\hline Varones (466) & $11^{\prime} 532$ (5'55) & $0 ’ 458\left(0^{\prime} 75\right)$ & $0^{\prime} 246\left(0^{\prime} 38\right)$ & 0'196 (0'18) & 2'659 (1'86) \\
\hline Mujeres (442) & $12^{\prime} 391\left(5^{\prime} 77\right)^{*}$ & 0’437 (0’65) & 0’265 (0'37) & $0 \prime 193\left(0^{\prime} 16\right)$ & 2'765 (1'86) \\
\hline
\end{tabular}

Nota: CDI: Cuestionario de Depresión Infantil; PNID-D (Inventario de Depresión de Nominación de Iguales: subescala de Depresión), PNID-F (idem, subescala de Felicidad), PNID-P (\{dem, subescala de Popularidad); CDCN-1: Cuestionario de Distorsiones Cognitivas para Niños.

* ANOVA unidireccional significativo $p<0$ 05. (1) El CDCN-1 se administró a 871 casos, 444 varones y 427 mujeres ( 37 fueron valores perdidos). 
según cada grupo de edad se visualiza en la Tabla 2. Las diferencias entre los grupos de edad son estadísticamente significativas para la medida CDI de depresión ( $F=7$ '194, $p=0$ '00001), siendo el grupo de 12 años el responsable de las mismas, en el sentido de exhibir las puntuaciones más bajas cuando se les compara con los jóvenes de 14, 15 y 16 años, respectivamente. Dicho resultado se pone de manifiesto con la prueba post hoc de Scheffé ( $p<0$ 0 05) estando, además, dotado de homogeneidad de la varianza (F de Bartlett $=2$ '033, no significativo).

En el caso del inventario PNID los diversos grupos se diferencian sólo en la escala de Felicidad ( $F=3^{\prime} 319, p=0$ '010), al contrario que para las subescalas de Depresión ( $F=1$ '259, $p=0$ '284) y Popularidad ( $F=1$ '206, $p=0$ '306). Las diferencias significativas en el caso de la subescala de Felicidad se establecen entre los grupos de 14 y 16 años, a favor de este último según la prueba de Scheffé ( $p<0$ 005).

El CDCN-1 muestra igualmente diferencias dotadas de significación estadística ( $F=2^{\prime} 707, p=0$ '029), pero no son atribuibles a ningún grupo en particular (Scheffé no significativo). Los diferentes grupos cumplieron el principio de homocedasticidad, salvo para las subescalas de Depresión y Felicidad del PNID.

\section{Diferencias en función del sexo y la edad}

En la Tabla 3 se recogen todos los promedios de cada una de las variables criterio en función del sexo para cada grupo de edad de forma separada. Las diferencias por sexo en los tres instrumentos aplicados no fueron estadísticamente significativas en ninguno de los cinco grupos de edad considerados, salvo para el caso de los varones de 14 años, quienes resultaron más populares que las mujeres como indican los datos procedentes de la subescala de Popularidad del PNID (F = 4'196, $p=0$ 0'041). Como ya se señaló cuando se consideró toda la muestra, puede apreciarse en cada categoría de edad que las mujeres presentan un promedio superior en el inventario CDI de depresión. Con todo, dentro de cada uno de estos niveles no se hallan diferencias relevantes desde un punto de vista estadístico para esta variable de salida.

A pesar de que estos guarismos no resulten dotados de significación, se observaron algunos productos según el sexo para cada grupo de edad que merecen reseñarse. Así, en las puntuaciones procedentes del inventario CDI, las mujeres de 14 y 16 años presentaron un promedio más pronunciado de respuestas en la dirección de la alteración anímica. En el caso de las distorsiones cognitivas

Tabla 2. Medias y desviaciones tipo (entre paréntesis) de las pruebas aplicadas a toda la población en función de la edad

\begin{tabular}{|c|c|c|c|c|c|}
\hline Edad & $C D I$ & PNID-D & PNID-F & PNID-P & $C D C N-1^{(1)}$ \\
\hline 12 Años (192) & $10^{\prime} 349\left(5^{\prime} 46\right)^{\star *}$ * & $0^{\prime} 387\left(0^{\prime} 69\right)$ & $0 ' 257\left(0^{\prime} 39\right)$ & $0,207\left(0^{\prime} 17\right)$ & 2'349 (1'62) \\
\hline 13 Años (195) & $11^{\prime} 538\left(6^{\prime} 19\right)$ & $0^{\prime} 469\left(0^{\prime} 75\right)$ & $0^{\prime} 263\left(0^{\prime} 39\right)$ & $0 ' 185\left(0^{\prime} 17\right)$ & 2'945 (1'91) \\
\hline 14 Años (192) & $12^{\prime} 447\left(5^{\prime} 46\right)$ & $0^{\prime} 520\left(0^{\prime} 86\right)$ & $0^{\prime} 174\left(0^{\prime} 24\right)^{*}$ & 0 '176 (0’17) & 2'804 (1'87) \\
\hline 15 Años (195) & $12^{\prime} 456\left(5^{\prime} 63\right)$ & $0^{\prime} 465\left(0^{\prime} 68\right)$ & $0^{\prime} 290\left(0^{\prime} 40\right)$ & 0'204 (0’17) & 2'673 (1'87) \\
\hline 16 Años (134) & $13^{\prime} 395\left(4^{\prime} 97\right)$ & 0'376 (0'39) & $0^{\prime} 309\left(0^{\prime} 45\right)$ & 0'205 (0'16) & 2'813 (2'02) \\
\hline
\end{tabular}

Nota: CDI: Cuestionario de Depresión Infantil; PNID-D (Inventario de Depresión de Nominación de Iguales: subescala de Depresión), PNID-F (ídem, subescale de Felicidad), PNID-P (ídem, subescala de Popularidad); CDCN-1: Cuestionario de Distorsiones Cognitivas para Niños.

" ANOVA unidireccional significativo $p<0$ '05 $y$ * * significativo para $p<0$ 01. (1) El CDCN-1 se administró a 871 casos, 444 varones y 427 mujeres ( 37 fueron valores perdidos). 
Tabla 3. Medias y desviaciones tipo (entre paréntesis) de las pruebas aplicadas a los escolares de 12 a 16 años

\begin{tabular}{|c|c|c|c|c|c|}
\hline & $\mathrm{CDI}$ & PNID-D & PNID-F & PNID-P & $\mathrm{CDCN}-1^{(1)}$ \\
\hline 12 años, $\mathrm{n}=192$ & $10^{\prime} 349\left(5^{\prime} 46\right)$ & 0'387 (0'69) & $0^{\prime} 258$ (0'39) & 0'207 (0'17) & $2^{\prime} 350$ (1'62) \\
\hline Varones (98) & $10^{\prime} 061\left(6^{\prime} 24\right)$ & $0^{\prime} 435\left(0^{\prime} 88\right)$ & $0^{\prime} 238\left(0^{\prime} 42\right)$ & $0,206\left(0^{\prime} 18\right)$ & $2^{\prime} 260\left(1^{\prime} 60\right)$ \\
\hline Mujeres (94) & $10^{\prime} 648\left(4^{\prime} 53\right)$ & $0^{\prime} 336\left(0^{\prime} 42\right)$ & $0^{\prime} 278\left(0^{\prime} 35\right)$ & $0^{\prime} 207\left(0^{\prime} 16\right)$ & 2'439 (1'64) \\
\hline 13 años, $n=195$ & $11^{\prime} 538(6 ' 19)$ & $0^{\prime} 470\left(0^{\prime} 75\right)$ & $0 ’ 264\left(0^{\prime} 39\right)$ & 0’185 (0’17) & 2'946 (1'91) \\
\hline Varones(101) & $11^{\prime} 128\left(5^{\prime} 68\right)$ & $0^{\prime} 425\left(0^{\prime} 68\right)$ & $0^{\prime} 280\left(0^{\prime} 41\right)$ & 0 '182 (0’17) & 2'851 (1'93) \\
\hline Mujeres (94) & $11^{\prime} 978\left(6^{\prime} 70\right)$ & 0’516 (0’81) & $0.245\left(0^{\prime} 36\right)$ & $0,187(0,16)$ & $3^{\prime} 044\left(1^{\prime} 89\right)$ \\
\hline 14 años, $n=192$ & $12 \prime^{\prime} 448\left(5^{\prime} 46\right)$ & $0 ’ 520\left(0^{\prime} 86\right)$ & $0^{\prime} 174\left(0^{\prime} 24\right)$ & $0 ' 177\left(0^{\prime} 17\right)$ & $2^{\prime} 804(1 ' 87)$ \\
\hline Varones (96) & $11^{\prime} 864\left(5^{\prime} 13\right)$ & $0^{\prime} 586\left(0^{\prime} 80\right)$ & $0^{\prime} 205\left(0^{\prime} 28\right)$ & $0^{\prime} 202\left(0^{\prime} 20\right)^{*}$ & $2^{\prime} 813\left(1^{\prime} 90\right)$ \\
\hline Mujeres (96) & $13^{\prime} 031\left(5^{\prime} 74\right)$ & $0^{\prime} 453\left(0^{\prime} 91\right)$ & $0^{\prime} 143\left(0^{\prime} 20\right)$ & 0'150 (0'14) & 2'795 (1'85) \\
\hline 15 años, $n=195$ & $12^{\prime} 456\left(5^{\prime} 63\right)$ & $0^{\prime} 466\left(0^{\prime} 68\right)$ & $0^{\prime} 290\left(0^{\prime} 40\right)$ & 0'204 (0'17) & $2^{\prime} 674(1 ' 87)$ \\
\hline Varones(101) & $12^{\prime} 158\left(5^{\prime} 06\right)$ & $0^{\prime} 480\left(0^{\prime} 83\right)$ & 0 '239 (0'37) & 0'199 (0'17) & 2'577 (1'79) \\
\hline Mujeres (94) & $12^{\prime} 776\left(6^{\prime} 20\right)$ & $0^{\prime} 449\left(0^{\prime} 48\right)$ & $0^{\prime} 344\left(0^{\prime} 42\right)+$ & $0,209\left(0^{\prime} 17\right)$ & $2^{\prime} 774\left(1^{\prime} 96\right)$ \\
\hline 16 años, $n=134$ & $13^{\prime} 396\left(4^{\prime} 97\right)$ & $0 ’ 377$ (0’39) & $0 ’ 309\left(0^{\prime} 45\right)$ & 0’206 (0’17) & $2^{\prime} 813\left(2^{\prime} 02\right)$ \\
\hline Varones (70) & $12 ' 814(5 ' 19)$ & $0^{\prime} 331\left(0^{\prime} 40\right)$ & $0^{\prime} 277\left(0^{\prime} 41\right)$ & $0 ’ 190\left(0^{\prime} 18\right)$ & $2^{\prime} 842\left(2^{\prime} 08\right)$ \\
\hline Mujeres (64) & $14^{\prime} 031$ (4'68) & $0^{\prime} 426\left(0^{\prime} 38\right)$ & $0^{\prime} 344\left(0^{\prime} 48\right)$ & $0^{\prime} 222\left(0^{\prime} 15\right)$ & $2^{\prime} 781$ (1'97) \\
\hline
\end{tabular}

Nota: CDI: Cuestionario de Depresión Infantil; PNID-D (Inventario de Depresión de Nominación de Iguales: subescala de Depresión), PNID-F (ídem, subescala de Felicidad), PNID-P (idem, subescala de Popularidad); CDCN-1: Cuestionario de Distorsiones Cognitivas para Niños.

* ANOVA unidireccional significativo $p<0$ '05 (las diferencias estadísticamente significativas son dentro de cada grupo de edad). ${ }^{(1)}$ El CDCN-1 se administró a 871 casos, 444 varones y 427 mujeres (37 fueron valores perdidos: 9 en el grupo de 12 años, 10 entre los de 13 años, 13 valores perdidos en el grupo de 14 años y 5 entre los de 15 años).

medidas por el CDCN-1, las mujeres de 13 años exhibieron mayor media, siendo los varones de 14 años quienes marcaron un mayor número de estos sesgos cognitivos. En las distintas subescalas del inventario PNID aparecieron algunas diferencias resaltables. En la subescala de Depresión, los varones de 12 y 14 años recibieron más indicadores que sus iguales femeninas y, en cambio, a los 13 años, las jóvenes superaron en puntuación a los varones. La subescala de Felicidad resultó más favorable entre las chicas de 14,15 y 16 años en comparación con los jóvenes de su mismo grupo de edad, siendo tendencial en el segundo caso.

$\mathrm{El}$ análisis factorial de la varianza $2 \mathrm{x}$ 5 para comprobar la contribución de las variables sexo y edad sobre las medidas aplicadas ratificó los resultados anteriores (Tabla 4). De esta manera, para el criterio de depresión (inventario CDI), se hallaron diferencias estadísticamente significativas en las variables del sexo y la edad, sin que se ofreciera un componente interactivo entre las mismas. En el caso de las distorsiones cognitivas (cuestionario CDCN-1), se comprobaron diferencias exclusivamente en el caso de la variable edad, probablemente porque la detección de las distorsiones dependa del desarrollo cognitivo de los sujetos. Como ya se señaló en el análisis aislado de esta variable en función de la edad, sí se detectan diferencias dependientes de la misma pero no aclara qué categoría marca un punto de inflexión, quizá porque las diferencias individuales se hallan detrás de los cambios madurativos generales.

\section{La relación entre las medidas}

El análisis de las correlaciones (Pearson, a dos colas) entre las diferentes 
Tabla 4. Análisis factorial de la varianza de las variables sexo y edad ( $2 \times 5)$ en el total de la muestra para las variables de medida CDI y $\mathrm{CDCN}-1$

\begin{tabular}{cllll}
\hline & & $F$ & $p$ & Variables de medida \\
\hline Efecto principal & & 6,84 & 0,0001 & \\
Variables predictivas & Sexo & 5,22 & 0,0220 & CDI \\
& Edad & 7,22 & 0,0001 & \\
Interacción & & 0,12 & 0,975 & \\
\hline Efecto principal & & 2,30 & 0,043 & CDCN-1 \\
Variables predictivas & Sexo & 0,07 & 0,3900 & \\
& Edad & 2,70 & 0,029 & \\
Interacción & & 0,18 & 0,945 & \\
\hline
\end{tabular}

Nota: CDI: Cuestionario de Depresión Infantil; CDCN-1: Cuestionario de Distorsiones Cognitivas para Niños.

variables de medida y la edad puede observarse en la Tabla 5. Este análisis es enormemente esclarecedor pues pone de relieve una relación significativa de 0'59 entre la puntuación total en depresión (CDI) y el número de distorsiones cognitivas (CDCN-1). El resto de los índices de correlación son bastante más modestos. Se observa una esperada relación entre la subescala de Depresión del PNID y el inventario CDI de 0'14, significativa por el tamaño de la población, a pesar de todas las dificultades respecto a la concordancia de fuentes que se dan habitualmente en la evaluación de la depresión (del Barrio, 1990). No obstante, no se acompaña del mismo resultados con las otras dos subescalas del inventario cita- do. La correlación del PNID de depresión con el cuestionario CDCN-1 también es significativo pero, nuevamente, el guarismo resultante es exiguo y producto del tamaño poblacional (0'12). Finalmente, dentro de la consistencia del inventario PNID, se da una correlación significativa y positiva entre las subescalas de Felicidad y Popularidad (0'48), acompañada de una correlación negativa y significativa entre la subescala de Depresión y de Popularidad (-0'10), pero no en el caso de la Felicidad y de Depresión.

Un resultado también reseñable se refiere a la correlación positiva y significativa entre el instrumento CDI de depresión y la edad, de casi 0'17. Nuevamente, se trata de una puntuación

Tabla 5. Correlaciones de Pearson (dos coles) entre las variables de medida y la edad para toda la población $(n=908)$

\begin{tabular}{|c|c|c|c|c|c|c|}
\hline & CDI & PNID1D & PNID2F & PNID3P & CDCN-1 & EDAD \\
\hline CDI & 1'0000 & & & & & \\
\hline PNID1D & $0^{\prime} 1472^{* *}$ & 1'0000 & & & & \\
\hline PNID2F & $-0 ’ 0204$ & $-0^{\prime} 0290$ & $1^{\prime} 0000$ & & & \\
\hline PNID3P & $-0 ’ 0612$ & $-0^{\prime} 1095^{* *}$ & $0^{\prime} 4833^{* *}$ & 1'0000 & & \\
\hline $\mathrm{CDCN}-1^{(1)}$ & $0^{\prime} 5915^{* \text { * }}$ & $0^{\prime} 1207^{* *}$ & 0,0073 & $-0^{\prime} 0098$ & $1 ' 0000$ & \\
\hline EDAD & $0^{\prime} 1686^{\star *}$ & 0'0041 & $0^{\prime} 0401$ & 0'0089 & 0’0492 & 1'0000 \\
\hline
\end{tabular}

Nota: CDI: Cuestionario de Depresión Infantil; PNID-D (Inventario de Depresión de Nominación de Iguales: subescala de Depresión), PNID-F (idem, subescala de Felicidad), PNID-P (idem, subescala de Popularidad); CDCN-1: Cuestionario de Distorsiones Cognitivas para Niños. (1) El CDCN-1 se administró a 871 casos (37 fueron valores perdidos). * Significativo $p<0$ '01, * Significativo $p<0$ '001 
determinada por el gran tamaño de la población, si bien, en los resultados que a continuación se presentarán, se adivina un paulatino aumento en las puntuaciones en el CDI en función de la edad. Sin embargo, este producto no se ve reforzado de forma tan clara con ninguna de las demás pruebas empleadas.

En el análisis de regresión múltiple (por pasos) partiendo del criterio de depresión proporcionado por el cuestionario CDI y las demás variables como predictivas, la ecuación resultante muestra que el principal elemento predictor de las puntuaciones de depresión recogidas en el inventario CDI es el resultante de las distorsiones cognitivas (cuestionario CDCN-1), explicando un $34^{\prime} 91 \%$ de la varianza $\left(\mathrm{T}=21^{\prime} 18, p==\right.$ 0'00001). El resto de las variables aporta una cantidad discreta de la explica- ción final (un $37^{\prime} 4 \%$ ). Así, la edad explica por sí sola un 1'6\% (los de más edad muestran puntuaciones más altas en el CDI) ( $\mathrm{T}=4{ }^{\prime} 87, p=0$ '00001); el PNID de Depresión explica un 0'59\% (también las puntuaciones más elevadas en esta subescala) ( $\mathrm{T}=3$ '07, $p=0$ '0021) y, por último, el sexo, concretamente el caso de las mujeres, alcanzando a representar tan sólo el 0 ' $3 \%$ de la varianza ( $\mathrm{T}=$ 2'28, $p=0$ '0223). Las subescalas de Felicidad y Popularidad no se incorporaron a la ecuación final.

Por tanto, como se aprecia en la Gráfica 1 y recopilando los productos de los diferentes estadísticos, las puntuaciones en sintomatología depresiva (CDI) ascienden lentamente de acuerdo con los años de los participantes. Además, parecen intervenir en alguna medida las diferencias de género, pero de forma poco relevante. En la parte

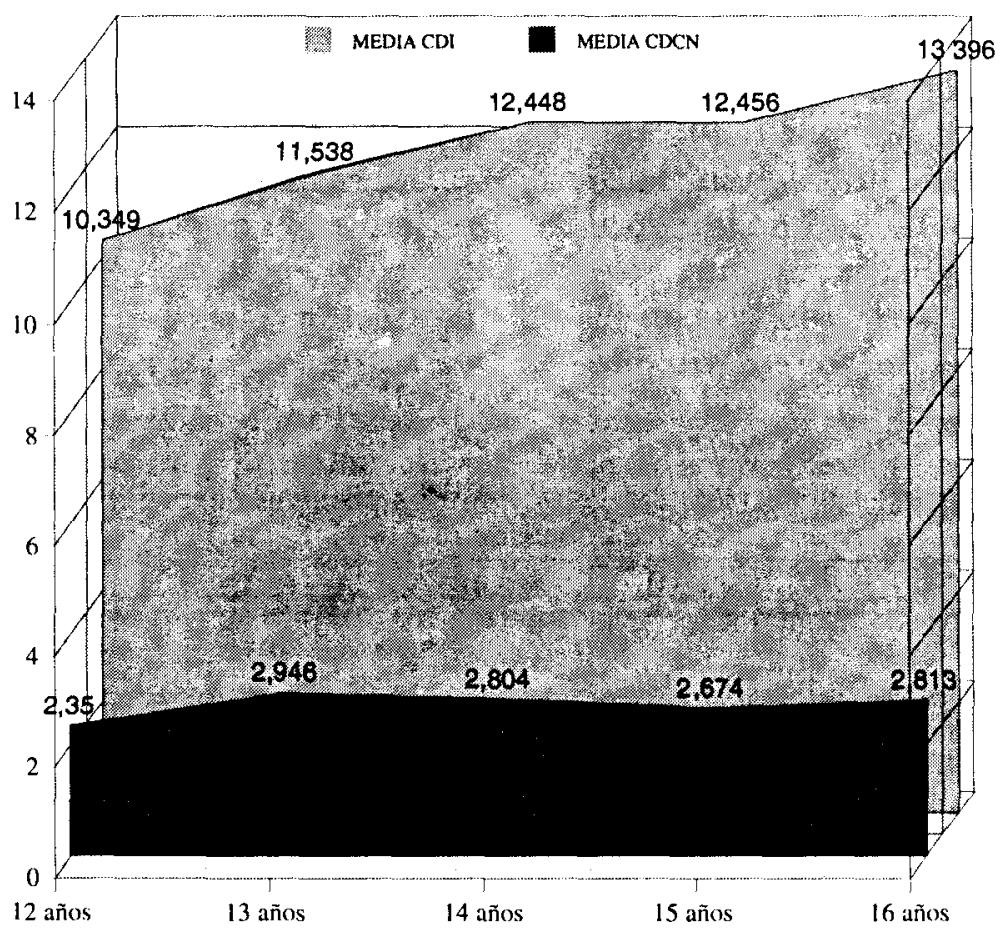

Gráfica 1. Puntuaciones medias en el inventario CDI (depresión) y el cuestionario CDCN-1 (distorsiones cognitivas) 
inferior de la gráfica se observa el promedio de distorsiones cognitivas en las que se comprueba un alza coincidente con uno de los mayores incrementos en las puntuaciones del CDI, esto es, en los trece años de edad. De resto, la puntuación en las distorsiones se mantiene más o menos constante para, de acuerdo con la puntuación del CDI, apreciarse a los 16 años una muy ligera subida en el promedio de los errores cognitivos.

Los jóvenes en riesgo o depresión posible y probable

Si se toman los casos que puntuaron por encima del punto de corte establecido para indicar respuestas o sintomatología significativa de depresión en el inventario CDI de depresión (mayor o igual a 19 puntos), se observa una nutrida presencia de jóvenes en dicha situación (107), representando un $11^{\prime} 78 \%$ (sobre los 908 participantes), $5^{\prime} 06 \%$ en el caso de los varones y un 6'71\% para las mujeres (la media de edad de este grupo es de 14'18 años, 1'31 de desviación típica). A pesar de darse un mayor número de casos entre las mujeres, las diferencias en las puntuaciones no se vieron influidas por el sexo ni en el inventario CDI ( $F=0$ '266, $p=0$ '606), en el CDCN-1 ( $F=0$ '281, $p$ = 0'596), o en las subescalas de Depresion del inventario PNID ( $F=1$ '342, $p$ $=0$ '249), de Felicidad ( $\mathrm{F}=0$ '058, $p=$ 0'810), o de Popularidad ( $F=0^{\prime} 957, p=$ 0'330).

Cuando se analiza la congruencia entre el cuestionario CDI (mayor o igual a 19 puntos) y el CDCN-1 (mayor o igual a 7), es decir, los jóvenes que superan los puntos de corte en ambas pruebas al tiempo, se halla un 2'87\% de los sujetos con puntuaciones significativas que se consideraron en riesgo de presentar o desarrollar un trastorno del estado de ánimo, o de depresión posible e incluso probable. De estos sujetos, 25 (sobre 871 casos), un $1^{\prime} 26 \%$ eran varones y un 1 '6\% mujeres. La media de edad en este grupo resultó similar a la anterior, 14'28 años (1'40 de desviación tipo). La distancia en el número de personas con puntuaciones significativas en función del sexo se recorta de forma llamativa hasta estar casi equiparados: 11 varones y 14 mujeres.

Los análisis unidireccionales de la varianza en este subgrupo de personas de riesgo no demuestran diferencias en el criterio CDI de depresión en cuanto al sexo $\left(F=0^{\prime} 208, p=0^{\prime} 652\right)$, eso sí, con medias muy elevadas entre los varones 24'18 puntos (5'13 de desviación) y las mujeres 25'35 (7'21 de desviación). Tampoco se observaron diferencias de género en el cuestionario de distorsiones cognitivas CDCN-1 $(F=$ 0'622, $p=0$ '438), ni en el PNID de Depresión ( $\left.F=0^{\prime} 755, p=0^{\prime} 735\right)$, de Felicidad ( $F=0^{\prime} 291, p=0$ '334) 0 de Popularidad ( $F=0$ '213, $p=0$ '217). Puede mencionarse que el promedio de distorsiones cognitivas en este grupo fue de 4'93 para los varones (2'03 de desviación) y de 4'71 para las mujeres (2'10 de desviación), mientras la subescala de depresión del inventario PNID no va de forma paralela con estos parámetros: 0'834 nominaciones medias entre los varones (1'37 de desviación) y 0’594 entre las mujeres (0'74 de desviación tipo).

\section{DISCUSIÓN}

Los resultados procedentes de otros estudios acerca dé la presencia de sintomatología depresiva en población española, aunque en un rango de edad diferente (de 8 a 12 años), tales como el de Polaino-Lorente y Doménech (1988), 
han puesto de manifiesto una prevalencia del $1{ }^{\prime} 8 \%$ de Trastorno Depresivo Mayor y del 6'4\% para el Trastorno Distímico, siendo la prevalencia global de Trastornos del estado de ánimo del 8 ' $2 \%$. Concretamente en Sevilla, con una submuestra de 993 sujetos (504 varones y 489 mujeres entre 9 y 11 años), la prevalencia de Trastorno Depresivo Mayor en el mencionado trabajo fue del 3'5\% y la de Trastorno Distímico del 6'9\%, obtenidas a través del CDI (en la fase de cribado) y posteriormente precisadas con los criterios del DSM-III (fase de diagnóstico). De este modo, la ciudad de Sevilla, sin una fácil explicación, obtuvo la cifra más alta de prevalencia de Depresión Mayor y la tercera en Trastorno Distímico frente al resto de las ciudades estudiadas. Si atendemos a la fase de filtrado de la investigación de Polaino-Lorente y Doménech (op.cit.), del conjunto de 1.211 sujetos, 471 superaron el punto de corte del inventario CDI, lo que representa un $38^{\prime} 89 \%$ con sintomatología depresiva (es decir, respuestas significativas al CDI). Este dato contrasta con el obtenido por Ezpeleta et al. (1990) en la ciudad de Barcelona con edades muy similares, pues la sintomatología significativa se observa en un $6^{\prime} 78 \%$ (igualmente dentro de la fase de cribado con el CDI).

En la presente investigación se obtiene un $11^{\prime} 78 \%$ de los sujetos que han puntuado por encima del punto de corte establecido para el inventario CDI, lo que representa un guarismo de cierta consideración si lo comparamos con el trabajo ya citado de Ezpeleta y colaboradores o con el margen del $15-17 \%$ obtenido por López Soler y López Pina (1998), teniendo en cuenta en este último caso, que se trata de población clínica (niños de 6 a 11 años). Pueden ofrecerse dos posibles comentarios para este resultado: por un lado, el antece- dente ya mencionado y particular en la ciudad de Sevilla de una mayor tendencia a la sintomatología y diagnósticos depresivos, por otro lado y probablemente más relevante, porque a diferencia de los tres trabajos anteriormente mencionados, esta investigación recoge una población preadolescente y adolescente. Como es sabido, es esperable un aumento en las cifras consideradas en estos márgenes de edad, especialmente cuando se ciñen a la sintomatología depresiva (Cicchetti y Toth, 1998; del Barrio, 1997; del Barrio et al., 1997). De hecho, los promedios en el inventario CDI aplicado en este estudio son similares a los alcanzados por Mestre et al. (1992) con un grupo de niños entre $14 \mathrm{y}$ 15 años: 11'4 de media global, 10'7 para los varones y 12'5 entre las mujeres. Así, resulta clarificador comprobar los promedios hallados en el CDI para cada una de las edades tomadas, apreciándose una tendencia lineal ascendente en función de la progresión de la edad (10'34, 11'53, 12'44, 12'45, 13'39 puntos), con un salto destacable en el paso de los 12 a los 13 años (gráfica 1). Esta línea ascendente en función de los años de los jóvenes ha sido señalada, de manera consensuada, por diversos autores (Frías et al., 1992; Gotlib y Hammen, 1996; Kazdin, 1990; Mestre et al., 1992; Nogueira y Monreal, 1990; PolainoLorente y García Villamisar, 1993; Reinherz, Frost y Pakiz, 1991).

Al hilo de lo mencionado, puede considerarse el estudio de Hankin et al. (1998) ya que se sugiere que el paso de la adolescencia media a la adolescencia tardía puede ser un momento crítico para generar vulnerabilidad a la depresión, dadas las altas puntuaciones en sintomatología depresiva en estas edades y los mayores riesgos que acompañan a esta etapa evolutiva: cambios de humor, aumento de conflictos con las figuras parentales o el mayor número de con- 
ductas de riesgo, elementos propiciadores de la concepción de la adolescencia como una etapa estresante (Arnett, 1999).

Tomando cada grupo de edad por separado no se observa una influencia de la variable sexo en ninguno de los criterios relacionados directamente con la depresión, ni tan siquiera, o precisamente menos, cuando se seleccionan los casos con puntuaciones significativas. Lo contrario sucede cuando se analiza el total de la población, coincidiendo así con la mayoría de estudios realizados con adolescentes, etapa en la que las diferencias de género parecen emerger en los grandes grupos (Frías et al., 1992; Hankin et al., 1998; Nolen-Hoeksema y Girgus, 1994; Reinherz et al., 1991). Este hecho lo han explicado Nolen-Hoeksema y Girgus (1994) según un modelo transaccional ecológico en el que los factores de riesgo a la depresión, similares en chicos y chicas, interaccionan con los trascendentes cambios de la etapa adolescente femenina (biológicos y psicosociales), originando una prevalencia depresiva superior en las mujeres que en los varones. Además, se ha determinado que ante la presencia de sintomatología depresiva los varones exhiben una mayor tendencia a las respuestas activas y distractoras, mientras las mujeres acostumbran a dar respuestas menos activas o más rumiadoras (un estilo autofocalizado y contemplativo) que perpetúa el estado de ánimo y que, posiblemente, tenga origen en la socialización diferencial recibida entre hombres y mujeres (Nolen-Hoeksema, 1987; 1991; Nolen-Hoeksema, Morrow y Fredrickson, 1993).

Otro de los objetivos de esta investigación era estudiar y establecer la relación entre los diferentes instrumentos aplicados. En primer lugar, en el caso del PNID, se observan promedios más bajos en la puntuación de la subescala de depresión de esta prueba que en el trabajo de Ezpeleta et al. (1990) ya mencionado, y equi- parable para la submuestra de Sevilla en la investigación nacional de PolainoLorente y Doménech (1988; 1990). En segundo lugar, diferentes aportaciones coinciden en las bajas correlaciones halladas entre dicho instrumento y el inventario CDI (entre 0'12 y 0'23) (Lefkowitz y Tesiny, 1985; Marujo y Da Silva, 1999; Polaino-Lorente y Doménech, 1990), e incluso sin correlación estadísticamente significativa (Ezpeleta et al., 1990). En el presente trabajo, la correlación entre dichos instrumentos resulta significativa aunque con una puntuación francamente modesta (0'14), de acuerdo con los estudios citados y, probablemente, efecto también del gran tamaño de la población participante.

Por tanto, consideramos que el inventario PNID no ha resultado muy útil y en la investigación sobre la evaluación de la depresión infantil se reitera la debilidad de esta prueba, ya que los compañeros parecen ser muy sensibles para detectar la popularidad, pero mucho menos en el caso de la tristeza (del Barrio, 1997). Si se analizan los datos, da la sensación de que el PNID acompaña a la puntuación del CDI a los 12 años, para luego ir descendiendo: $r=0$ '25 ( $p<0$ 0'001) a los 12 años; $r=0$ '16 (n.s.) a los 13; $r=0$ '08 (n.s.) a los 14; $r=0$ '09 (n.s.) a los $15 \mathrm{y}$, sorprendentemente, $\mathrm{r}=0$ '25 a los 16 años $(p<$ 0 001). Si junto a estos datos, se analiza la correlación de esta subescala de depresión del PNID con respecto a la edad, (correlaciones de 0'38; 0'47; 0'52; 0'46 y 0'37, para cada nivel de edad, respectivamente), se puede observar un pico a los 14 años, esperable tendencia al alza en la relación depresión-edad, para luego descender.

En suma, se trata de una distribución simétrica que tal vez indique que el uso de este inventario no sea aconsejable más allá de los 14 años, señal probable de que la sintomatología pueda modificarse o de que comienza a ser menos observable, en 
favor de una posible internalización de los síntomas. Los promedios obtenidos en la subescala de depresión del PNID, para los chicos es más elevado que para las chicas, salvo en el grupo de 13 años (hecho sin una clara explicación). Es posible que sea debido a que los varones manifiesten a sus compañeros la sintomatología depresiva de forma más externalizada que las mujeres (Nolen-Hoeksema y Gírgus, 1994), al menos en este período de edad, o bien que exista un proceso más lento de internalización de la sintomatología en el varón que en la mujer en sus trayectorias evolutivas. La importancia de la percepción de popularidad por los compañeros y la sintomatología depresiva, es igualmente apoyada si observamos la correlación hallada entre las subescalas de depresión y popularidad (de casi -0'11), de manera que los depresivos serían los menos populares. Sin embargo, no se detecta un resultado comparable con respecto a la subescala de felicidad. Parece, al tiempo, que la principal correlación de la subescala de felicidad es con la de popularidad (0'48). Quizás es más fácil observar la felicidad entre los más populares, quienes no son precisamente los depresivos, o tal vez porque se observan mejor las muestras de felicidad entre los más populares. Estos datos nos sugieren la importancia de la percepción del otro en estos niveles de edad y en este tipo de variables, como ha indicado la literatura relacionada con el estatus sociométrico y el desajuste emocional (Cole y Carpentieri, 1990; Gotlib y Hammen, 1996; Patterson y Stoolmiller, 1991). Cole y Carpentieri (1990), utilizando un procedimiento de nominación entre iguales, encuentran que los niños rechazados presentaron las mayores puntuaciones en depresion y, coherentemente, los niños populares las menores. Para Patterson y Stoomiller (1991) estas variables son significativas si media con las malas relaciones entre iguales $y$, para
Gotlib y Hammen (1996), es la depresión la generadora del rechazo interpersonal.

De mayor interés resulta el cuestionario CDCN-1 como un complemento muy adecuado del inventario CDI gracias a una correlación bastante aceptable entre sus puntuaciones (0'59), teniendo presente, como ya se ha dicho, que son pruebas concebidas desde un mismo modelo, aunque, y esto es lo interesante, muy diferentes en cuanto al contenido de sus ítemes (Polaino-Lorente, Buceta y Maldonado, 1986). Por tanto, la consideración conjunta de ambos instrumentos representaría una forma de validez del constructo de la depresión desde la perspectiva cognitiva o hipótesis de la primacía (Clark y Beck, 1997). Los resultados referidos por Bas (1987a), creador del cuestionario $\mathrm{CDCN}-1$, coinciden plenamente con los productos obtenidos en la presente investigación. Así, la media poblacional hallada es de 2'71 puntos, es decir, de casi 3 distorsiones cognitivas (polarización, abstracción selectiva, maximización, etcétera) de un total de 11 posibles y la mencionada correlación de 0'59. Bas (1987a) obtuvo una correlación de 0 '58 (con el CDI) y un promedio de 2'87 distorsiones (para una muestra de 58 sujetos).

Estos resultados son acordes con los encontrados por otros autores sobre los errores cognitivos y la sintomatología depresiva (Haley et al., 1985; Kendall, 1990; Leitenberg et al. 1986). De hecho, Kendall (1990) encuentra una correlación significativa entre las puntuaciones obtenidas en el CDI y las distorsiones cognitivas (en relación con las percepciones negativas que realiza el sujeto del ambiente, así como la desvaloración no. realista de sí mismo y sus actuaciones), avalando la consideración de una red nomológica de la depresión infantil.

En la distribución de las puntuaciones se descubre un salto cuantitativo que aparece en los 13 años, casi con una media 
de 3 distorsiones cognitivas, pudiendo hacerse la lectura de la edad como un indicador indirecto de la aparición de determinados sesgos en el procesamiento de la información personalmente relevante. Como hallaron Nolen-Hoeksema, Seligman y Girgus (1992) en un estudio longitudinal con niños de 8 años, el estilo cognitivo depresógeno sólo se constituyó en un predictor de la sintomatología depresiva en aquellos niños de mayor edad (10 a 12 años). Cuando los niños son pequeños, el número de eventos negativos son los mejores predictores de sintomatología depresiva. Por consiguiente, cabe especular que el incremento de las capacidades cognitivas en el niño, confiere una mayor estabilidad a sus cogniciones que hacen que adquieran una capacidad predictiva sobre la sintomatología depresiva. En otras palabras, la mayor posibilidad valorativa-interpretativa (y de sesgos) en el joven es un nefasto aliado ante los eventos (vitales) negativos acaecidos y de la modificación del estado de ánimo que tiene lugar tras éstos.

Esto significa que el elemento distorsiones cognitivas se emplea en una doble vertiente: por un lado, como un ingrediente que surge (o se consolida) con la maduración general de cualquier individuo pero, de otro lado, como una variable contribuyente en los desórdenes del estado de ánimo. Se asume pues, una perspectiva dimensional que hace esperable cierto grado de sesgos cognoscitivos en una persona, pero el estado de ánimo depresivo incrementa su aparición (hipotesis de la gravedad, de la exclusividad y del procesamiento selectivo), y las distorsiones cognitivas agravan el humor insertas ya en un círculo vicioso (Teasdale, $1983 ; 1988$ ). Junto a estos elementos se añade la idea de Beck de que estos procesos cognitivos son comunes también a otros trastornos (sobre todo de ansiedad, los más estudiados junto con la depresión), cambiando únicamente el contenido de los productos (1976, pág. 90; Clark y Beck, 1997), aspecto que no hemos puesto a prueba en este trabajo (hipótesis de la especificidad del contenido).

Cuando se usan conjuntamente ambos instrumentos con puntuaciones significativas se registra una media próxima a las 5 distorsiones cognitivas (CDCN-1) y una presencia de sintomatología depresiva de relieve (CDI), generando una cifra equivalente a los diferentes trabajos de prevalencia puntual basados en criterios diagnósticos al uso (APA, 1995; Boyd y Weissman, 1985; Cross-National Collaborative Group, 1992; Friedman y Thase, 1995; Schramm, 1998; Weissman et al., 1993), por ello aludimos en los resultados la consideración de depresión posible e incluso probable, ya que no se contó con una entrevista diagnóstica estructurada o la aplicación de los criterios de diagnóstico internacionales.

Siguiendo esta idea, no se trata de otorgar a las distorsiones cognitivas un papel causal sobre la depresión, supuesto que, por otro lado, no puede garantizarse con este diseño, sino que éstas desempeñan, al menos, un factor concomitante y agravante de las alteraciones anímicas (Beck, 1988; 1991; 1997; Teasdale, 1988), además de otorgarle a las cogniciones negativas un papel primario en la experiencia depresiva (Clark y Beck, 1997); hecho que en la actualidad queda experimentalmente demostrado (Vázquez y Cameron, 1997; Zuckerman, 1999). En el presente trabajo observamos que, en efecto, las distorsiones cognitivas tienen un papel predictivo sobre la sintomatología depresiva de casi el $35 \%$ de la varianza explicada. Además, como se ha dicho, guardan relación con los momentos de mayór desarrollo cognoscitivo en el niño-joven, hecho corroborado por el alza en el promedio de distorsiones cognitivas que acompañan a la sintomatología depresiva (en los 13 
años), pero también, como se mencionó más arriba, por el momento cronológico en el que el PNID, como prueba basada en la observación de conductas, empieza a no tener utilidad ante la internalización de las manifestaciones depresivas (sobre los 14 años).

El estudio prospectivo de Lewinsohn, Allen, Gotlib y Seeley (1999) confirma la asociación del humor disfórico con el pensamiento disfuncional para el grupo de adolescentes con historia de Trastorno Depresivo Mayor, lo que sugiere, como se ha dicho, que las distorsiones cognitivas derivan y exarceban el estado de ánimo depresivo. Las diferentes investigaciones llevadas a cabo y revisadas por Abramson et al. (1999) basadas en la sintomatología depresiva (análogos clínicos), en la llamada depresión por desesperanza y en el Trastorno Depresivo Mayor, más el mencionado trabajo de Lewinsohn et al. (y los anteriores de este grupo), nos lleva a sugerir que, probablemente, los jóvenes que puntúan de forma significativa tanto en el CDI como en el CDCN-1 estén en riesgo de desarrollar (o desarrollan ya) un cuadro depresivo, con la salvedad de que, en la población objeto de este estudio, no se ha llevado a cabo un diagnóstico formal de Trastorno del estado de ánimo. En otras palabras, si asumimos los supuestos del modelo de Teasdale avalados por Lewinsohn et al. (1999), el hecho de hallar una correlación significativa entre el CDCN-1 y el CDI, una predicción de éste a partir de las distorsiones más un grupo de jóvenes que superen claramente los puntos de corte de ambas pruebas, puede sugerir que la coincidencia con puntuaciones elevadas en ambas medidas son claros indicios de un elevado riesgo (vulnerabilidad) para la depresión o, tal vez, de estar desarrollando un primer episodio depresivo (depresión posible o tal vez probable).

Sin embargo, no debe olvidarse que esta investigación se caracteriza por una estructura transversal y no nos hemos centrado en analizar diversas variables posiblemente relevantes como diferentes condiciones sociodemográficas (por ejemplo Reinherz, Giaconia, Hauf, Wasserman, y Silverman, 1999), de ciertas variables biológicas, o al constatado influjo del estrés, como subrayan los trabajos recientes del Instituto de Investigación de Oregón (USA). Así, por ejemplo, Monroe, Rohde, Seeley y Lewinsohn (1999) y Lewinsohn et al. (1999) han dedicado esfuerzos en comprobar el papel de ciertos estresores de intensidad como marcadores del primer episodio de depresión mayor, siendo el más relevante entre los adolescentes, una ruptura en una relación sentimental. De hecho, atendiendo a las variables consideradas en la predicción de la sintomatología depresiva (CDI) del presente estudio, se supera ligeramente el $37 \%$ de explicación de la varianza, guarismo a todas luces insuficiente pero concentrado, como se ha dicho, en las distorsiones cognoscitivas.

En cualquier caso, consideramos que el manejo conjunto de los instrumentos CDNC-1 y CDI pueden ser de utilidad en los centros escolares siempre que conlleve una finalidad de cribado o de detección precoz de posibles casos. Además, esta combinación es atractiva por su fácil manejo, porque parte de un mismo modelo teórico y porque ayudaría a marcar los contenidos cognitivos susceptibles de trabajarse tanto en los casos ya corroborados, en aquellos con puntuaciones limítrofres o en aquellos otros sin guarismos significativos. De este modo, sería muy interesante desarrollar programas de intervención sobre los contextos escolares y familiares, como medios socializadores de transmisión de cogniciones erróneas (Cicchetti y Toth, 1998; Dodge, 1993; Doménech, 1988; Olmedo, del Barrio y Santed, 1998; Stark et al., 1993) y directamente sobre las propias cogniciones infantiles que configuran 
determinadas estructuras de conocimiento, tales como las distorsiones cognitivas, estilos atribucionales, y que guían el procesamiento depresógeno de la información del niño, funcionando como elementos de vulnerabilidad hacia la adquisición de sintomatología depresiva. En tal camino ha ido los trabajos de Asarnow y Bates (1988) que han puesto el acento de su investigación en la búsqueda del papel desempeñado por los distintos tipos de pensamientos negativos en la depresión de los adolescentes.

Ya sabemos que a estas variables cognitivas resulta arriesgado otorgarles un papel definitivo de causa, consecuencia o de concomitante de la depresión, pero desde el modelo de Teasdale $(1983 ; 1988)$ su aparición (debidas o no al estado de ánimo) contribuyen a perpetuar el humor depresivo. Aunque el volumen de información en la literatura es claramente superior en el caso de las atribuciones (Polaino-Lorente, 1988b; Wierzbicki, Peterson, Kaslow, Alloy, y Abramson, 1984), quizá sea necesario considerar otras variables implicadas en el procesamiento de la información del depresivo, como el caso de las distorsiones cognitivas apuntadas por Beck (1976; Clark y Beck, 1997; Vázquez y Cameron, 1997) y contempladas en el presente estudio ante la escasez de datos al respecto en nuestro contexto científico.

Finalmente, como se desprende de los anteriores párrafos, esta investigación tiene las limitaciones propias de un trabajo meramente exploratorio. En primer lugar, porque debe reiterarse que los instrumentos aplicados no proporcionan un diagnóstico, ni tan siquiera al utilizarlos de forma conjunta. En todo caso, son una ayuda adicional al mismo, además de que no pueden representar más que una forma de recoger la sintomatología depresiva (que hemos equiparado a un número significativo de respuestas a los autoinformes).
En segundo lugar, porque sería preciso desarrollar un procedimiento más amplio en el que tengan cabida variables más relevantes (sociales, familiares, biológicas, paso en el que estamos en el momento en que se redactan estas líneas) así como analizar otros elementos del procesamiento de la información y, a ser posibles, no limitados a autoinformes (Vázquez y Cameron, 1997).

En tercer y último lugar, sería más relevante para poner a prueba el modelo cognitivo de Beck en su parte descriptiva o causal (Clark y Beck, 1997) por medio de un diseño longitudinal y prospectivo, es decir, en el que se parta de diagnósticos formales de Trastornos del estado de ánimo y sin que tamaño de las muestras limiten la generalización de los resultados.

\section{REFERENCIAS BIBLIOGRÁFICAS}

Abramson, L.Y., Alloy, L.B., Hogan, M.E., Whitehouse, W.G., Donovan, P., Rose, D.T., Panzarella, C., y Raniere, D. (1999). Cognitive Vulnerability to Depression: Theory and Evidence. Journal of Cognitive Psychotherapy: An International Quarterly, 13, 5-20. Abramson, L.Y., Alloy, L.B., Metalsky, G.I., Joiner, T.E., y Sandín, B. (1997). Teoría de la Depresión por Desesperanza: Aportaciones Recientes. Revista de Psicopatología y Psicología Clínica, 2, 211-222.

American Psychiatric Association (APA) (1995). DSM-IV. Manual Diagnóstico y Estadístico de los Trastornos Mentales. Barcelona: Masson, S.A. (original en inglés: 1994).

Anderson, J.C., Williams, S., McGee, R., y Silva, P.A. (1987). DSM-III Disorders in Preadolescent Children. Prevalence in a Large Sample from the General Population. Archives of General Psychiatry, 44, 69-76. 
Angold, A., y Rutter, M. (1992). Effects of Age and Pubertal Status on Depression in a Large Clinical Sample. Development and Psychopathology, 4, 5-28.

Arnett, J.J. (1999). Adolescent storm and stress, reconsidered. American Psychologist, 54, 317-326.

Asarnow, J.R., y Bates, S. (1988). Depression in Child Psychiatric Inpatients: Cognitive and Attributional Patterns. Journal of Abnormal Psychology, 16, 601-616.

Baker, F.M. (1991). A Contrast: Geriatric Depression versus Depression in Younger Age Groups. Journal of the National Medical Association, 83, 340-344.

Bas Ramallo, F. (1987a). Distorsiones Cognitivas en Adolescentes Deprimidos. II Congreso de Evaluación Psicológica. Madrid. Publicado en el Giornale Italiano di Scienzia e Terapia del Comportamiento, 22-23, 109-114, 1989.

Bas Ramallo, F. (1987b). Cuestionario de Distorsiones Cognitivas para Niños y Adolescentes (CDCN-1). Centro de Psicología «Bertrand Russel». Madrid.

Bas Ramallo, F. (1996). Intervención en Crisis en Niños y Adolescentes Deprimidos en el Contexto Escolar. Psicología Educativa, 11, 55-65.

Bas Ramallo, F., y Andrés Navia, V. (1994). Terapia Cognitivo-Conductual de la Depresión: Un Manual de Tratamiento. Madrid: Fundación Universidad-Empresa.

Beck, A.T. (1976). Cognitive Therapy and the Emotional Disorders. New York: International Universities Press, Inc. Beck, A.T. (1988). Terapia Cognitiva, Terapia Conductual, Psicoanálisis y Farmacoterapia, un Continuo Cognitivo. En M.J. Mahoney y A. Freeman, Cognición y Psicoterapia (pp 349-371). Barcelona: Paidós. (Publicación original en inglés: 1985).

Beck, A.T. (1991). Cognitive Therapy. A 30-Year Retrospective. American Psychologist, 46, 368-375.
Beck, A.T. (1997). Terapia Cognitiva: Pasado, Presente y Futuro. En M.J. Mahoney (Ed.), Psicoterapias Cognitivas y Constructivistas. Teoría, Investigación y Práctica (pp 49-61). Bilbao: Desclée de Brouwer.

Beck, A.T., Rush, A.J., Shaw, B.F., y Emery, G. (1983). Terapia Cognitiva de la Depresión (3 ed.). Bilbao: Desclee de Brouwer. (Publicación original en inglés: 1979).

Boyd, J.H., y Weissman, M.M. (1985). Epidemiología. En E.S. Paykel (Coord.), Psicopatología de los Trastornos Afectivos (pp 179-204). Madrid: Pirámide. (Publicación original en inglés: 1982).

Bragado, C., Carrasco, I., Sánchez Bernardos, M.L., Bersabe, R.M., Loriga, A., y Monsalve, T. (1995). Prevalencia de los Trastornos Psicopatológicos en Niños y Adolescentes: Resultados Preliminares. Clínica y Salud, 6, 67-82.

Cantwell, D.P., y Carlson, G.A. (1987). Trastornos Afectivos en la Infancia y la Adolescencia. Barcelona: Martínez Roca (original en inglés: 1983).

Carlson, G.A., y Cantwell, D.P. (1980). A Survey of Depressive Symptoms, Syndrome and Disorder in a Child Psychiatric Population. Journal of Child Psychology and Psychiatry, 21, 19-25.

Carlson, G.A., y Kashani, J.H. (1988). Phenomenology of Major Depression from Childhood Through Adulthood: Analysis of Three Studies. American Psychiatric Association, 145, 1222-1225.

Cicchetti, D., y Toth, S. (1998). The Development of Depression in Children and Adolescents. American Psychologist, 53, 221-241.

Clark, D.A., y Beck, A.T. (1997). El Estado de la Cuestión en la Teoría y la Terapia Cognitiva. En I. Caro (Comp.), Manual de Psicoterapias Cognitivas (pp 119-129). Barcelona: Paidós.

Cohen, P., Cohen, J., Kasen, E., Velez, C.N., Hartmark, C., Johnson, J., Rojas, 
M., Brook, J., y Streuning, E.L. (1993). An Epidemiological Study of Disorders in Late Childhood and Adolescence-I. Age and Gender Specific Prevalence. Journal of Child Psychology and Psychiatry, 34, 849-865.

Cole, D.A., y Carpentieri, S. (1990). Social Status and the Comorbidity of Child Depression and Conduct Disorder. Journal of Consulting and Clinical Psychology, 58, 748-757.

Compas, B.E., Oppedisano,G., Connor, J., Gerhardt, C.A., Hinden, B.R., Achenbach, T.M., y Hammen, C. (1997). Gender Differences in Depressive Symptoms in Adolescence: Comparison of National Samples of Clinically Referred and Nonreferred Youths. Journal of Consulting and Clinical Psychology, 65, 617-626.

Costello, E.J., Costello, A.J., Edelbrock, C., Burns, B., Dulcan, M.K., Brent, D., y Janiszewski, S. (1988). Psychiatric Disorders in Pediatric Care. Archives of General Psychiatry, 45, 1107-1116.

Cross-National Collaborative Group (1992). The Changing Rate of Major Depression. Cross-National Comparisons. Journal of the American Medical Association, 268, 3098-3105.

Del Barrio, V. (1990). Situación Actual de la Evaluación de la Depresión Infantil. Evaluación Psicologica-Psychological Assessment, 6, 171-209.

Del Barrio, V. (1997). Depresión Infantil. Concepto Evaluación y Tratamiento. Barcelona: Ariel.

Del Barrio, V., y Moreno, C. (1996). Evaluación de la Depresión y la Ansiedad Infantil. Ansiedad y Estrés, 2, 131-158. Del Barrio, Moreno, C., y López, R. (1997). Ecology of Depression in Spanish Children. European Psychologist, 2, 18-27.

Dodge, K.A. (1993). Social-Cognitive Mechanisms in the Development of Conduct Disorder and Depression. Annual Review of Psychology, 44, 559-584.
Doménech, E. (1988). Prevención de la Depresión Infantil. En A. PolainoLorente, Las Depresiones Infantiles (pp 217-251). Madrid: Morata.

Doménech E., y Moreno, M. (1995). Depresión en la Edad Preescolar. En E. Doménech (Ed.), Actualizaciones en Psicopatología Infantil (0-5 años). Barcelona: PPU.

Ezpeleta, L. (1988). Epidemiología de la Depresión Infantil en el Municipio de Barcelona. Revista de Psiquiatría de la Facultad de Medicina de Barcelona, 15, 255-268.

Ezpeleta, L., Polaino- Lorente, A., Doménech, E., y Doménech, J. (1990). Peer Nomination Inventory of Depression: Characteristics in a Spanish Sample. Journal of Abnormal Child Psychology, 18, 373-391.

Fleing, J.E., y Offord, D.R. (1990). Epidemiology of Childhood Depressive Disorders: A Critical Review. Journal of the American Academy of Child and Adolescence Psychiatry, 29, 571-580.

Frías, D., Mestre, V., Del Barrio, V., y García-Ros, R. (1992). Estructura Familiar y Depresión Infantil. Anuario de Psicología, 52, 121-131.

Friedman, E.S., y Thase, M.E. (1995). Trastornos del Estado de Ánimo. En V.E. Caballo, G. Buela-Casal y J.A. Carrobles (Dirs.), Manual del Psicopatología (Vol. 1, pp 619-681). Madrid: Siglo XXI.

Gladstone, T.R., y Kaslow, N.J. (1995). Depression and Attributions in Children and Adolescents: a Meta-Analytic Review. Journal of Abnormal Child Psychology, 23, 597-606.

Gotlib, I.H., y Hammen, C.L. (1996), Psychological aspects of depression. New York: Wiley.

Haley, G.M., Fine, S., Marriage, K., Moretti, M.M., y Freeman, R.J. (1985). Cognitive Bias and Depression in Psychiatrically Disturbed Children and Adolescents. Journal of Consulting and Clinical Psychology, 53, 535-537. 
Hankin, B.L., Abramson, L.Y., Moffitt, T.E., Silva, P.A., y McGee, R. (1998). Development of Depression from Preadolescence to Young adulthood: Emerging Gender Differences in a 10-Year Longitudinal Study. Journal of Abnormal Psychology, 107, 128-140.

Harrison, R.P., Beck, A.T., y Buceta, J.M. (1984). Terapia Cognitiva de la Depresión: Una Introducción al Desarrollo Histórico. Los Conceptos y Procedimientos Fundamentales de la Alternativa Terapéutica de Beck. Revista de Psicología General y Aplicada, 39, 623646.

Instituto Andaluz de Estadística (1992). Censo de Población de Andalucía. Resultados Provisionales. Autor. Joiner, T.E., Blalock, J., y Wagner, K. (1999). Preliminary Examination of Sex Differences in Depressive Symptoms among Adolescent Psychiatric Inpatients: the Role of Anxious Symptoms and Generalized Negative Affect. Journal of Clinical Child Psychology, 28, 211-219.

Karel, M.J. (1997). Aging and Depression: Vulnerability and Stress across Adulthood. Clinical Psychology Review, 17, 847-879.

Kashani, J.H., y Carlson, G.A. (1987). Seriously Depressed Preschoolers. American Journal of Psychiatry, 3, 348-350.

Kashani, J.H., Holcomb, W.R., y Orvaschel, H. (1986). Depression and Depressive Symptoms in Preschool Children from the General Population. American Journal of Psychiatry, 143, 1138-1143.

Kashani, J.H., y Ray, J. (1987). Major Depression with Delusional Features in a Preschool Age Child. Journal American Academy of Child and Adolescent Psychiatry, 26, 110-112.

Kashani, J.H., y Ray, J.S., Gabrielle, P.D., y Carlson, M.D. (1984). Depression and Depressive-Like States in PreschoolAge Children in a Child Development
Unit. American Psychiatric Association, 141, 1397-1402.

Kazdin, A. (1990). Childhood Depression. Journal of Child Psychology and Psychiatry, 31, 121-160.

Kazdin, A., Rodgers, A., y Colbus, D. (1986). The Hopelessness Scale for Children: Psychometric Characteristics and Concurrent Validity. Journal of Consulting and Clinical Psychology, 54, 241-245.

Kendall, P.C. (1990). Cognitive Deficit or Cognitive Distorsion in Childhood Depression. Journal of Abnormal Child Psychology, 18, 225-270.

Kovacs, M. (1992). Children's Depression Inventory, CDI. Toronto: Multi-Health Systems, Inc.

Kovacs, M. (1985). The Natural History and Curse of Depressive Disorders. Psychiatric Annals, 15, 387-389.

Lefkowitz, M., y Tesiny, E. (1981). Assessment of Childhood Depression. Journal of Consulting and Clinical Psychology, 48, 43-50.

Lefkowitz, M., y Tesiny, E. (1985). Depression in Children: Prevalence and Correlates. Journal of Consulting Clinical Psychology, 53, 647-656.

Leitenberg, H., Yost, L.M., y Carroll-Wilson, M. (1986). Negative Cognitive Errors in Children: Questionnaire Development Normative Data, and Comparisons between Children with and without Self-Reported Symptoms of Depression, Low-Steem, and Evaluation Anxiety. Journal of Consulting and Clinical Psychology, 54, 528-536.

Lewinsohn, P.M., Allen, N.B., Gotlib, I.H., Seeley, J.R. (1999). First Onset Versus Recurrence of Depression: Differential Processes of Psychosocial Risk. Journal of Abnormal Psychology, 108, 483-489. Lewinsohn, P.M., Gotlib, I.H., y Seeley, J.R. (1997). Depression-Related Psychosocial Variables: are they Specific to Depression in Adolescent?. Journal of Abnormal Psychology, 106, 365-375. 
Lewinsohn, P. M., Hops, H., Roberts, R. E., Seeley, J.R., y Andrews, J.A. (1993). Adolescent Psychopathology: I Prevalence and Incidence of Depression and other DSM-III-R Disorder in High School Students. Journal of Abnormal Psychology, 102, 133-144.

Lewinsohn, P. M., Roberts, R. E., Seeley, J.R., Rohde, P., Gotlib, I., y Hops, H. (1994). Adolescent Psychopathology: II Psychosocial Risk Factors for Depression. Journal of Abnormal Psychology, 103, 302-315.

López Sóler, C., y López Pina, J. (1998). La Depresión en la Infancia desde la Perspectiva de las Taxonomías Empíricas. Revista de Psicopatología y Psicología Clínica, 3, 95-102.

Marujo, H.A., y Da Silva, A.L. (1999). Divergencias entre Evaluadores en la Depresión Infanto Juvenil. Revista Iberoamericana de Diagnóstico y Evaluación Psicológica, (1), 65-83.

McGee, R., Feehan, M., Williams, S., Partridge, F., Silva, P., y Kelly, A.B. (1990). DSM-III Disorders in a Large Sample of Adolescents. Journal of the American Academy of Child and Adolescent Psychiatry, 29, 611-619.

McNeil, J.K., y Harsany, M. (1989). An Age Difference View of Depression. Canadian Psychology, 30, 608-615.

Mestre, V., Frías, D., García-Ros, R., y del Barrio, V. (1992). Prevalencia de la Depresión en la Infancia y la Adolescencia. En V. Mestre (Ed.), La Depresión en Población Adolescente Valenciana. Un Estudio de Seguimiento desde la Infancia a la Adolescencia. Valencia: Consellería de Sanitat i Consum.

Monroe, S.M., Rohde, P., Seeley, J.R., y Lewinsohn, P.M. (1999). Life Events and Depression in Adolescence: Relationship Loss as a Prospective RiskFactor for First Onset of Major Depressive Disorder. Journal of Abnormal Psychology, 108, 606-614.
Nogueira, M., y Monreal, P. (1990). Depresión Infantil y Variables Demográficas. En E. Doménech y A. PolainoLorente (Eds), Epidemiología de la Depresión Infantil. Barcelona: Espaxs.

Nolen-Hoeksema, S. (1987). Sex Differences in Unipolar Depression: Evidence and Theory. Psychological Bulletin, 101, 259-282.

Nolen-Hoeksema, S. (1991). Responses to Depression and Their Effects on the Duration of Depressive Episodes. Journal of Abnormal Psychology, 100, 569582.

Nolen-Hoeksema, S., y Girgus, J. (1994). The Emergence of Gender Differences in Depression During Adolescence. Psychological Bulletin, 115, 424-443.

Nolen-Hoeksema, S., Morrow, J., y Fredrickson, B.L. (1993). Response Styles and the Duration of Episodes of Depressed Mood. Journal of Abnormal Psychology, 102, 20-28.

Nolen-Hoeksema, S., Seligman, M.E., Girgus, J. (1992). Predictors and Consequences of Childhood Depressive Symptoms: a 5 Years Longitudinal Study. Journal of Abnormal Psychology, 101, 405-422.

Olmedo, M., del Barrio, V., y Santed, M.A. (1998). Prevención de Emociones Negativas en la Adolescencia: Valoración de Técnicas Cognitivo-Conductuales. Revista de Psicopatología y Psicología Clínica, 3, 121-138.

Patterson, G.R., y Stoolmiller, M. (1991). Replications of a Dual Failure Model for Boys'Depressed Mood. Journal of Consulting and Clinical Psychology, 59, 491-498.

Petersen, A. C., Sarigiani, P.A., y Kennedy, R.E. (1991). Adolescent Depression: Why More Girls?. Journal of Youth and Adolescence, 20, 247-271.

Polaino-Lorente, A. (1988a). Las Depresiones Infantiles. Madrid: Morata.

Polaino-Lorente, A. (1988b). Aportaciones y Contradicciones de la Psicología 
de la Atribución en la Explicación de las Depresiones Infantiles. En A. Polaino-Lorente, Las Depresiones Infantiles (pp 252-274). Madrid: Morata.

Polaino-Lorente, A. (1996). Trastornos Afectivos en la Infancia. En M.C. Luciano Soriano (Dir.), Manual de Psicología Clínica. Infancia y Adolescencia (pp 640-664). Valencia: Promolibro.

Polaino-Lorente, A. (1995). Los Trastornos Afectivos en la Infancia. Las Depresiones Infantiles. En J. Rodríguez Sacristán (Ed), La Psicopatología del Niño y el Adolescente (pp 655-688). Sevilla: Universidad de Sevilla.

Polaino-Lorente, A., Buceta Fernández, J., y Maldonado, C. (1986). La Evaluación del Progreso Terapéutico en la Depresión: Necesidad de los Registros Multidimensionales. Revista de Psicología General y Aplicada, 41, 339-357.

Polaino-Lorente, A., y Doménech, E., (1988). La Depresión en los Niños Españoles de 4 de E.G.B. Barcelona: GEIGY.

Polaino- Lorente, A., y Doménech, E., (1990). Utilidad y Limitaciones de la Escala PNID en la Evaluación Diagnóstica de la Depresión Infantil. Revista de Psiquiatría de la Facultad de Medicina de Barcelona, 17, 12-22.

Polaino, A., y García-Villamisar, D.A. (1993). La depresión infantil en Madrid. Un Estudio Epidemiológico. Madrid: Editorial Ac.

Reinherz, H.Z., Frost, A.K., y Pakiz, B. (1991). Chanching Faces: Correlates of Depressive Symptoms in Late Adolescence. Family and Community Health, 14, 52-63.

Reinherz, H.Z., Giaconia, R.M., Hauf, A.M.C., Wasserman, M.S., y Silverman, A.B. (1999). Major Depression in the Transition to Adulthood: Risks and Impairments. Journal of Abnormal Psychology, 108, 500-510.

Rutter, M., Tizard, J., y Whitemore, K. (1970). Education, Health and Behaviour. London: Longman.
Schramm, E. (1998). Psicoterapia Interpersonal. Barcelona: Masson. (Publicación original en alemán: 1996).

Seligman, M.E., Kaslow, N.J., Alloy, L.B., Peterson, C., Tanenbaum, R. L., y Abramson, L.Y. (1984). Attributional Style and Depressive Symptoms among Children. Journal of Abnormal Psychology, 93, 235-238.

Stark, K.D., Humphrey, L., Laurent, J., Livingston, R., y Christopher, J. (1993). Cognitive, Behavioral, and Family Factors in the Differentiation of Depressive and Anxiety Disorders during Childhood. Journal of Consulting and Clinical Psychology, 61, 878-886.

Teasdale, J.D. (1983). Negative Thinking in Depression: Cause, Effect or Reciprocal Relationship? Advances in Behaviour Research and Therapy, 5, 3-25.

Teasdale, J.D. (1988). Cognitive Vulnerability to Persistent Depression. Cognition and Emotion, 2, 247-274.

Vázquez, C., y Cameron, C. (1997). Taxonomía Cognitiva, Psicopatología y Psicoterapias Cognitivas. En I. Caro (Comp.), Manual de Psicoterapias Cognitivas (pp 53-70). Barcelona: Paidós.

Weiss, B., Weisz, J.R., Politano, M., Carey, M., Nelson, W.M., y Finch, A.J. (1992). Relations among Self-Reported Depressive Symptoms in Clinic Referred Children versus Adolescents. Journal of Abnormal Psychology, 101, 391-397.

Weissman, M.M., Bland, R., Joyce, P.R., Newman, S., Wells, J.E., y Wittchen, H.V. (1993). Sex Differences in Rates of Depression: Cross-National Perspectives. Journal of Affective Disorders, 29, 77-84. Wierzbicki, M.E.P., Peterson, C, Kaslow, N. J., Tanembaum, R.L., Alloy, L.B., y Abramson, L.Y. (1984). Attributional Style and Depressive Symptoms among Children. Journal of Consulting and Clinical Psychology, 43, 390-397.

Zuckerman, M. (1999). Vulnerability to Psychopathology. A Biosocial Model. Washington: APA. 